\title{
WATER WAVES ON SLOPING BEACHES
}

\section{HANS LEWY}

1. Introduction. Consider the time-dependent velocity potential $\phi(x, y, t)$ of the motion of an incompressible inviscid liquid in two space dimensions $x, y$. The Newtonian equations and the condition of incompressibility lead to the following mathematical model: Laplace's equation

$$
\frac{\partial^{2} \phi}{\partial x^{2}}+\frac{\partial^{2} \phi}{\partial y^{2}}=0
$$

and Bernoulli's law

$$
\frac{\partial \phi}{\partial t}+\left(\left(\frac{\partial \phi}{\partial x}\right)^{2}+\left(\frac{\partial \phi}{\partial t}\right)^{2}\right) / 2+g y+p=\text { const. }
$$

relating the pressure $p$ and the gravity potential $g y$ per unit mass to the velocity potential; here the $x$-axis is horizontal, the $y$-axis vertical and upwards and $g$ is the modulus of gravity acceleration. At the boundary of air and water the pressure is supposed to have a constant value $p_{0}$; thus (1.2) relates implicitly the surface elevation $y$ to the velocity potential. A considerable simplification is introduced by assuming the motion to be small of first order so that in (1.2) the quadratic terms may be cancelled. Then the motion becomes a small perturbation of the equilibrium position in which the surface will be thought of as given by $y=0$. For small elevations of the surface one concludes from (1.2) that, but for terms of higher order,

$$
y=y(x, t)=-\frac{\partial \phi}{\partial t}(x, 0, t) / g,
$$

where the constant on the right hand has been absorbed in a properly modified $\phi$. To this equation is added the condition that a particle at the surface remains at the surface; that is (using the "substantial" time derivative of (1.2)),

$$
d y(x, t) / d t=-\frac{\partial^{2} \phi}{\partial t^{2}} / g=\partial \phi / \partial y,
$$

An address delivered at the invitation of the Committee to Select Hour Speakers for Far Western Sectional Meetings before the Pasadena meeting of the Society on November 24, 1945; received by the editors May 15, 1946. 
where terms of second order, such as $\left(\partial^{2} \phi / \partial x \partial t\right)(\partial \phi / \partial x)$, have been dropped in comparison with $\partial^{2} \phi / \partial t^{2}$.

Among these infinitesimal motions the periodic motions are of particular interest. Set

$$
\phi(x, y, t)=\phi_{1}(x, y) \cos \nu t-\phi_{2}(x, y) \sin \nu t,
$$

where $\nu>0$ is the so-called circular frequency. From (1.1) we take

$$
\left(\partial^{2} / \partial^{2} x+\partial^{2} / \partial^{2} y\right) \phi_{1}(x, y)=0, \quad\left(\partial^{2} / \partial^{2} x+\partial^{2} / \partial^{2} y\right) \phi_{2}(x, y)=0
$$

$$
y=y(x, t)=(\nu / g) \phi_{1}(x, 0) \sin \nu t+(\nu / g) \phi_{2}(x, 0) \cos \nu t ;
$$

from (1.3.1)

$$
\begin{aligned}
\left(\nu^{2} / g\right)\left(\phi_{1}(x, 0) \cos \nu t-\right. & \left.\phi_{2}(x, 0) \sin \nu t\right) \\
& =\left(\partial \phi_{1} / \partial y\right) \cos \nu t-\left(\partial \phi_{2} / \partial y\right) \sin \nu t
\end{aligned}
$$

Special cases occur if one of the two functions $\phi_{1}, \phi_{2}$, say $\phi_{2}$, is taken identically zero. Then the crests or troughs and the nodes of the oscillation retain their fixed positions in space. Crests or troughs of the surface elevation occur where $\partial \phi_{1}(x, 0) / \partial x=0$, nodes where $\phi_{1}(x, 0)$ $=0$. Such a periodic oscillation is called a "standing wave" in contrast to the more general case of a "progressive wave," where the crests and troughs and the nodes change position with time. For the horizontal velocity component $v_{\text {nod }}$ of the surface nodes we write (1.6) in the form $\tan \nu t=-\phi_{2}(x, 0) / \phi_{1}(x, 0)$ or $\nu t=-\arctan \left(\phi_{2} / \phi_{1}\right)$; differentiation with respect to $t$ yields

$$
v_{\text {nod }}=\nu /\left(\left(d \arctan \phi_{1} / \phi_{2}\right) / d x\right) .
$$

Similarly the horizontal component $v_{\text {or }}$ of the crest or trough velocity is found to be

$$
v_{\text {or }}=\nu /\left(\left(d \arctan \frac{d \phi_{1}}{d x} / \frac{d \phi_{2}}{d x}\right) / d x\right) .
$$

Both $\phi_{1}(x, y)$ and $\phi_{2}(x, y)$ are solutions of the same problem

$$
\partial^{2} \phi / \partial x^{2}+\partial^{2} \phi / \partial y^{2}=0
$$

throughout the liquid,

$$
\nu^{2} \phi(x, 0) / g=\frac{\partial \phi}{\partial y}(x, 0)
$$

at the surface. 
One of the fascinating problems concerning waves is the discovery of the influence of the depth of the water on the shape of the wave profile. Take first the classical case of infinite depth. To (1.7) and (1.8) is added the condition that the magnitude of the motion decreases to zero as $y \rightarrow-\infty$. Out of the manifold of all harmonic functions the real and imaginary parts of

$$
\exp \left(-i(x+i y) \nu^{2} / g\right)
$$

are taken as solutions $\phi_{1}$ and $\phi_{2}$ of (1.7) satisfying the boundary condition (1.8). With these is constructed the progressive wave $\phi(x, y, t)=R \exp \left(-i(x+i y) \nu^{2} / g-i t\right)=\exp \left(\nu^{2} y / g\right) \cos \left(\nu^{2} x / g+\nu t\right)$, which is periodic in $x$ as well as in $t$; the $x$-period is $2 \pi g / \nu^{2}$, the $t$-period $2 \pi / \nu$. The wave profile travels unchanged in form in the direction of diminishing $x$ as $t$ increases; the velocity of the profile is $v_{\text {nod }}=v_{\text {or }}=-g / \nu$ and depends therefore on the frequency.

Airy was the first to give a treatment of the infinitesimal gravity waves in a channel of constant finite depth; his results are the foundation of our knowledge on gravity waves. Again as in the case of infinite depth, the profile of the progressive wave moves, unchanged in form, with constant velocity. But the wave velocity depends on both the frequency and the depth of the channel, and so does the space period of the wave. Airy's formulae show with great clarity the passage from waves in water of finite depth to the well known limit theory of shallow water waves in whose treatment the dependence of the pressure and velocity on the depth is assumed rather than derived.

The problem whose solution forms the object of this paper is the construction of progressive water waves when the depth, instead of being a constant, has a constant slope. This problem was first attacked by E. T. Hanson ${ }^{1}$ in 1926 . He assumed the angle of the bottom with the surface to be of the form $\pi /(2 q)$ with integral $q$, and gave the expression of one standing wave $\phi_{1}(x, y)$ in terms of exponential functions, without, however, constructing the progressive wave which requires the derivation of a second standing wave $\phi_{2}(x, y)$. M. Miche ${ }^{2}$ quotes an explicit form of this second function $\phi_{2}$, which he calls "houle," to distinguish it from $\phi_{1}$, called "clapotis." For the derivation

\footnotetext{
${ }^{1}$ E. T. Hanson, Proc. Roy. Soc. London. Ser. A vol. 111 (1926) p. 491.

${ }^{2} \mathrm{M}$. Miche, Mouvements ondulatoires de la mer en profondeur constance ou décroissante, 1945, p. 88. This booklet also contains a most interesting report of various investigations of $M$. Miche, relating to the breaking of waves. See also $M$. Miche, Annales des ponts et chaussées, 1944, pp. 25-78, 131-164, 270-292, 369-406. Unfortunately, by the time of printing of the present paper, the author had had no opportunity to consult these latter articles.
} 
of his formula he refers the reader to a paper to appear in the Journal de mathématiques pures et appliquées; also his result is restricted to angles of form $\pi /(2 q)$.

We shall give in this paper an explicit representation in closed form for a progressive wave for all angles $p \pi /(2 q)$ between bottom and surface, provided $p$ and $q$ are integers, $p$ is odd, and $p<2 q$. The functions involved in our formulae are exponential functions and exponential integrals of certain special types. (Unfortunately, the number of individual terms occurring in the solution becomes large as $p$ or $q$ become large.) In the course of our investigation we are led to the discovery of a strange and recondite relationship of these waves with the quadratic reciprocity law of number theory. In fact, this law appears as an elementary consequence of the continuous dependence of the finite standing wave on the angle between surface and bottom. We do not go here more deeply into the discussion of certain numbertheoretical problems immediately suggested by this relationship, nor do we investigate the continuity of the waves as functions of the angle, for which there appears to exist a more direct approach than through our explicit expressions for these waves. A further topic left untouched is the extension of the principles of construction discussed here to the study of the three-dimensional wave problem where the wave crests are no longer parallel to the shore.

The complexity of our formulae for the waves for large $p$ or $q$ and the scarcity of suitable tables of exponential integrals make the evaluation of these waves a problem of considerable difficulty. It seems important to compare the "exact" theory of infinitesimal waves with other approximations such as the shallow water theory, in order to gauge the differences of the results furnished by the various theories. These problems, for angles $\pi /(2 q)$, are attacked by M. Miche (loc. cit.) and by J. J. Stoker in a paper under press.

2. Reformulation of the standing wave problem. The units of length and time will now be chosen so that

$$
g=1 \text { and } \nu=1 .
$$

Set

$$
x+i y=z=e^{i \theta}
$$

and let

$\beta=\pi p /(2 q), \quad p$ odd and relatively prime to $q$, and $0<p<2 q$.

Denote by $S_{\beta}$ the angular sector $0>\theta>-\beta$. The problem of the standing wave for $S_{\beta}$ consists in finding a harmonic function $\phi$ in $S_{\beta}$ satis- 
fying the following boundary and limit conditions, to be stated first for the case $p \leqq q$ :

1. $\partial \phi / \partial y-\phi=0$ on $y=0, x>0$;

2. The normal derivative $\partial \phi / \partial n=0$ on the bottom of $S_{\beta}$;

3. $\phi-A \cos x-B \sin x \rightarrow 0$ for suitable constants, not both zero, $A$ and $B$, as $x \rightarrow \infty, y=0$;

4. The square of the particle velocity, $(\partial \phi / \partial x)^{2}+(\partial \phi / \partial y)^{2}$, remains bounded as $z$ tends to $\infty$ in $S_{\beta}$;

5. The flow through any vertical section

$$
\int_{-x \tan \beta}^{0} \frac{\partial \phi}{\partial x}(x, y) d y
$$

remains bounded as $x \rightarrow \infty$.

To these conditions must be added one concerning the behavior of $\phi$ and its derivatives at the shore $(0,0)$. We shall see that there is one standing wave where

6a. $\phi$ and $\partial \phi / \partial x, \partial \phi / \partial y$ remain bounded as $(x, y)$ tends to $(0,0)$, and another where

6b. $\phi$ behaves like $\log \left(x^{2}+y^{2}\right)$ near $(0,0)$.

A solution of the above problem, if it exists, may be considered as the real part of an analytic function $W(z)$ in $S_{\beta}$. It can easily be concluded that $W(z)$ remains bounded as $z \rightarrow \infty$. For this purpose consider the conjugate harmonic $\psi$. Since the normal derivative of $\phi$ vanishes on the bottom of $S_{\beta}$, so does the tangential derivative of the conjugate $\psi$, whence $\psi$ is a constant there. But

$$
\begin{aligned}
\psi(x, 0)-\psi(x,-x \tan \beta) & =\int_{-x \tan \beta}^{0} \frac{\partial \psi}{\partial y}(x, y) d y \\
& =\int_{-x \tan \beta}^{0} \frac{\partial \phi}{\partial x}(x, y) d y,
\end{aligned}
$$

so that $\psi(x, 0)$ remains bounded at the surface as $x \rightarrow \infty$, by virtue of the 5 th condition. From the 4 th condition it follows furthermore that $|W(z)|$ can grow at most like const. $|z|$ as $z \rightarrow \infty$ within $S_{\beta}$. But then the Phragmén-Lindelöf principle applies and yields the boundedness of $|W(z)|$ for $z \rightarrow \infty$ in $S_{\beta}$. Indeed, for the application of this principle it is not at all necessary to demand the condition 4 which states that $\left|W^{\prime}(z)\right|$ remains bounded as $z \rightarrow \infty$; but it suffices to demand, besides $1,2,3$,

$4^{\prime} .|W(z)|<\exp |z|^{1+\epsilon}$ as $z \rightarrow \infty$ in $S_{\beta}$, for all $\epsilon>0$, together with

$5^{\prime} .|W(z)|$ remains bounded on the surface as $z=x \rightarrow \infty$. For then the principle states that $|W(z)|$ remains bounded as $z \rightarrow \infty$ in 
$S_{\beta}$. If $p<q$, then $4^{\prime}$ and $5^{\prime}$ imply 4 and 5 . For it is then possible to extend $W(z)$ analytically by reflection on the bottom of $S_{\beta}$ where condition 2 holds; and the double angle $2 \beta=\pi p / q$ is still less than $\pi$. In the process of extension any bound for the absolute value of $W(z)$ on the surface of $S_{\beta}$ becomes a bound for $|W(z)|$ on the bottom of $S_{2 \beta}$. Accordingly, $|W(z)|$ remains bounded on the boundary of $S_{2 \beta}$ as $z \rightarrow \infty$ and furthermore $|W(z)|<\exp |z|^{1+e}$ as $z \rightarrow \infty$ in $S_{2 \beta}, \epsilon>0$. Moreover, $W(z)$ can be continued analytically across $y=0$, since this is true for the function $F(z) \equiv i d W /(d z)-W$ whose real part vanishes on $y=0$. A bound of $|F(z)|$ for $y \leqq 0$ becomes at the same time a bound of $|F(z)|$ for $y>0$. Let $|W(z)| \leqq M$ for $y \leqq 0, x$ sufficiently large; then $|d W / d z| \leqq M /|y|$ by Cauchy's integral theorem. Describe a horizontal-vertical square $Q$ of side 4 about a point $x_{0}$ of the surface as center and consider $F_{1}(z)=F(z)\left(z-x_{0}-2\right)\left(z-x_{0}+2\right)$. On $Q$ we have $\left|F_{1}(z)\right| \leqq 54 M$; hence we conclude for $|y| \leqq 2$ that $\left|F_{1}\left(x_{0}+i y\right)\right|$ $\leqq 54 M$, where $\left|F\left(x_{0}+i y\right)\right| \leqq 54 M /(|i y-2||i y+2|) \leqq 14 M$, and, finally, from the definition of $F(z)$ and the bound $M$ for $|W(z)|$ $=\left|W\left(x_{0}+i y\right)\right|$ that $|d W / d z| \leqq 15 M$ for $x_{0}$ sufficiently large, $-2 \leqq y$ $\leqq 0$. For $y<-2$ Cauchy's estimate gives immediately $|d W / d z| \leqq M$, so that $|d W / d z|$ remains bounded in $S_{\beta}$. Hence 4 and 5 are consequences of $1,2,3,4^{\prime}, 5^{\prime}$.

If $p$ is no longer less than $q$, but $p<2 q$, we shall still be able to exhibit a solution $W(z)$ of the wave problem satisfying $1,2,3,4^{\prime}, 5^{\prime}$; but we shall then show explicitly that $|W(z)|$ remains bounded on the bottom of $S_{\beta}$ as $z \rightarrow \infty$, a fact which, as we have just seen, follows from the Phragmén-Lindelöf principle for $p<q$.

Let $\lambda$ be an arbitrary complex number of modulus 1 , and let $\partial \phi / \partial s$ stand for the derivative in the direction of the vector $\lambda$. Then

$$
\partial R W / \partial s=\partial \phi / \partial s=R \partial W / \partial s=R\{\lambda d W /(d \lambda s)\}=R\{\lambda d W / d z\} .
$$

Accordingly, the condition 1 may be rephrased as

$$
R\{(i d / d z-1) W(z)\}=0
$$

on $y=0$. Similarly for condition 2 : set

$$
\epsilon^{1 / 2}=e^{-\beta i}, \quad \epsilon=e^{-2 \beta i}=e^{-p x i / q} ;
$$

then this condition becomes

$$
R\left\{i \epsilon^{1 / 2} d W / d z\right\}=0 \text { on } \theta=-\beta=-\pi p /(2 q) .
$$

We furthermore see that reflection on the bottom of $S_{\beta}$ yields

$$
R\{(-i \epsilon d / d z-1) W(z)\}=0 \text { on } \theta=-2 \beta=-\pi p / q \text {. }
$$


3. Derivation of an ordinary differential equation. Let $f(\xi)$ and $g(\xi)$ be polynomials with real coefficients. Then, by (2.1),

$$
R\{f(d / d z)(i d / d z-1) W(z)\}=0
$$

on $y=0$, and by (2.4),

$$
\begin{aligned}
g(\partial / \partial s) R\{(-i \epsilon d / d z-1) W(z)\} \\
\quad=R\{g(\epsilon d / d z)(-i \epsilon d / d z-1) W(z)\}=0
\end{aligned}
$$

on $\theta=-2 \beta$. Suppose we succeed in solving the identical equation

$$
f(\xi)(i \xi-1) \equiv g(\epsilon \xi)(-i \epsilon \xi-1) ;
$$

then

$$
f(d / d z)(i d / d z-1) W(z)
$$

becomes a function $E(z)$, regular within the sector $0>\theta>-2 \beta$, and whose real part equals zero on the boundary of $S_{2 \beta}$ (with the possible exception of the origin). Now all such functions $E(z)$ are known explicitly. Our problem thus becomes that of solving an ordinary differential equation

$$
f(d / d z)(i d / d z-1) W(z)=E(z)
$$

for an appropriate $E(z)$, and of choosing from the manifold of its solutions one, $W(z)$, such as to satisfy the boundary conditions (2.1) and (2.3) as well as certain conditions concerning the behavior of $W(z)$ near $z=0$ and $z=\infty$.

\section{Derivation of $f(\xi)$.}

LEMMA. There exist polynomials $f(\xi)$ and $g(\xi)$ satisfying (3.3) and of degree $q-1$. They are uniquely determined by requiring the degree to be $q-1$ and $f(0)=1$. Furthermore $f(\xi) \equiv g(\xi)$.

The following proof is valid only if $p$ is an odd number. This is the reason why our explicit construction of waves fails if $p$ is even.

Let

$$
\begin{aligned}
& f(\xi)=a_{0}+a_{1} \xi+\cdots, \\
& g(\xi)=b_{0}+b_{1} \xi+\cdots .
\end{aligned} \quad a_{0}=1,
$$

The identity to be solved is

$$
(i \xi-1)\left(1+a_{1} \xi+\cdots\right)=(-i \epsilon \xi-1)\left(b_{0}+b_{1} \xi+\cdots\right) .
$$

Compare coefficients and find 


$$
\begin{aligned}
1 & =b_{0}, \\
i-a_{1} & =-i \epsilon b_{0}-b_{1} \epsilon \\
\ldots \ldots & \ldots . . . \\
i a_{n-1}-a_{n} & =-i \epsilon^{n} b_{n-1}-b_{n} \epsilon^{n}
\end{aligned}
$$

The imaginary part of the left-hand side is $a_{n-1}$ and may be considered known from the preceding equation of the recursion. The imaginary part of the right-hand side involves $b_{n-1}$ and $b_{n}$. Now $b_{n-1}$ also may be assumed known from the previous equation. Consequently $b_{n}$ is determined by equating imaginary parts, provided $I\left(\epsilon^{n}\right) \neq 0$, that is, for all $n$ with $0<n<q$, since $p$ and $q$ have no common divisors. Once $b_{n}$ is known, we take the value of $a_{n}$ from the same equation by equating real parts. Thus the recursion furnishes in a unique way all coefficients $a_{n}, b_{n}$ for $n<q$. There is a further condition to be satisfied to establish (4.2), namely, (4.3) for $n=q$, which becomes

$$
i a_{q-1}-a_{q}=-i \epsilon^{q} b_{q-1}-b_{q} \epsilon^{q} .
$$

Since $\epsilon^{q}=-1$ for odd $p$, this implies

$$
a_{q-1}=b_{q-1}
$$

if this is so, we may assume all further coefficients $a_{q}, b_{q}, a_{q+1}, b_{q+1}, \ldots$ to vanish. Now observe that $a_{0}=b_{0}=1$, and that the $n$th recursion formula (4.3) may be satisfied by setting $a_{n}=b_{n}$, provided it is true that $a_{n-1}=b_{n-1}$. Indeed, the uniquely determined solution of the recursion formulae can be found from the recursion

$$
a_{n}\left(1-\epsilon^{n}\right)=a_{n-1} i\left(1+\epsilon^{n}\right) .
$$

This gives for $a_{n}$ the nonvanishing value

$$
a_{n}=a_{n-1} i\left(1+\epsilon^{n}\right) /\left(1-\epsilon^{n}\right),
$$

which is indeed real since the vector $1-\epsilon^{n}$ is perpendicular to $1+\epsilon^{n}$. This holds provided $\epsilon^{n} \neq 1$, that is, as long as $n p / q \neq 2 k$ with integral $k$; hence certainly for odd $p$ and $0<n<q$. Notice that (4.5) can also be written as

$$
a_{n}=a_{n-1} \cot (n p \pi / 2 q)=\prod_{k=1}^{n} \cot (k p \pi / 2 q), \quad \text { for } n \geqq 1 .
$$

If we had permitted $p$ to be even, then $q$ would necessarily be odd and we would have $\epsilon^{q}=1$; in this event (4.4) would yield 


$$
i a_{q-1}\left(1+\epsilon^{q}\right)=2 a_{q-1} i=a_{q}-b_{q} \neq 0,
$$

which cannot be solved with real $a_{q-1}, a_{q}, b_{q}$.

Thus we have found

$$
\begin{array}{ll}
f(\xi) \equiv g(\xi) \equiv 1, & \text { for } q=1, \\
f(\xi) \equiv g(\xi) \equiv 1+\sum_{n=1}^{q-1} i^{n} \xi^{n} \prod_{k=1}^{n} \frac{1+\epsilon^{k}}{1-\epsilon^{k}}, & \text { for } q \geqq 2 .
\end{array}
$$

LEMMA. Let $q>1$. The roots of $f(\xi)$ are

$$
-i \epsilon,-i \epsilon^{2}, \cdots,-i \epsilon^{q-1} \text {; }
$$

they are conjugate to and, as a set, identical with

$$
i / \epsilon, i / \epsilon^{2}, \cdots, i / \epsilon^{q-1} \text {. }
$$

In fact, since by (3.3)

$$
(i \xi-1) f(\xi)=(-i \epsilon \xi-1) f(\epsilon \xi),
$$

it follows that the root of the right-hand side $\xi=i / \epsilon$ must also be a root of $f(\xi)$ since it is distinct from $-i$. But as all coefficients of $f(\xi)$ are real, the conjugate $-i \epsilon$ is also a root of $f(\xi)$. Substitute $\xi=-i \epsilon$ on the right; then either $\epsilon^{2}=-1$ or $f\left(-i \epsilon^{2}\right)=0$. Hence either $q=2$, or $-i \epsilon^{2}$ is a root of $f(\xi)$. In the latter case put $\xi=-i \epsilon^{2}$ in (4.6) and find either $\epsilon^{3}=-1, q=3$, or $f\left(-i \epsilon^{3}\right)=0$. Repeating this procedure we find that the complete set of roots of $f(\xi)$ is given by $\xi=-i \epsilon^{n}$, $n=1, \cdots, q-1$. Hence

$$
f(\xi)=c \prod_{n=1}^{q-1}\left(\xi+i \epsilon^{n}\right)
$$

where

$$
c^{-1}=f(0) / c=\prod_{n=1}^{q-1}\left(i \epsilon^{n}\right)=i^{q-1} \epsilon^{q(q-1) / 2}=(-1)^{(p-1)(q-1) / 2},
$$

in view of (2.2). Thus

$$
f(\xi)=(-1)^{(p-1)(q-1) / 2} \prod_{n=1}^{q-1}\left(\xi+i \epsilon^{n}\right) .
$$

Upon combining factors which contain conjugate roots of $f(\xi)$, we have

$$
f(-i)=(-1)^{(p-1)(q-1) / 2}(-i+i \epsilon)\left(-i+i \epsilon^{q-1}\right) \cdots .
$$

If $q$ is odd, we have $(q-1) / 2$ such pairs of factors; if $q$ is even, we have 
$(q-2) / 2$ such pairs and the factor $-i+i \epsilon^{q / 2}$. Now suppose first that both roots entering such a pair (for example, $-i \epsilon,-i \epsilon^{q-1}$ ) have positive real part. Then the argument of the product of such a pair remains the same if both roots are replaced by +1 ; that is, the argument of the product of such a pair of factors is $\pi / 2$. If, on the other hand, both roots involved lie on the left of the imaginary axis, the argument of such a product remains the same if both roots are replaced by -1 , which gives precisely $-\pi / 2$; and no root $i / \epsilon^{n}= \pm i$, since $0<n<q$.

Accordingly, for odd $q$,

$$
f(-i)=(-1)^{(p-1)(q-1) / 2}(-i)^{\alpha(p, q)} e^{-\pi i(q-1) / 4} r,
$$

where $r>0$ and $\alpha(p, q)$ is the number of roots of $f(\xi)$ with positive real part. It is easily seen that this formula still holds good for even $q$. To determine $r$, we note that

$$
\begin{aligned}
r^{2}=\left|\prod_{1}^{q-1}\left(1-\epsilon^{n}\right)\right|^{2} & =\prod_{1}^{q-1}\left(1-\epsilon^{n}\right)\left(1-\epsilon^{-n}\right) \\
& =\left|\frac{d}{d x}\left(\frac{x^{2 q}-1}{x+1}\right)\right|_{x=1}=q,
\end{aligned}
$$

so that

$$
f(-i)=(-1)^{(p-1)(q-1) / 2}(-i)^{\alpha(p, q)} e^{-\pi i(q-1) / 4} q^{1 / 2} .
$$

The number of terms with positive real part in the set $i / \epsilon^{j}$ is the same as the number of terms among $\epsilon^{-i}$ with negative imaginary parts. Note that

$$
e^{\pi i p j / q} \cdot e^{\pi i p(q-j) / q}=-1 .
$$

Hence, if $e^{\pi i p j / q}$ has a negative imaginary part, so does $e^{\pi i p(q-j) / q}$. Thus, for odd $q, \alpha$ is even and $(-i)^{\alpha(p, q)}=(-1)^{N(p, q)}$, where $N(p, q)$ is defined (for $q$ odd or even) by

$$
\begin{aligned}
N(p, q)= & \text { number of terms with negative imaginary parts in the } \\
& \text { set } e^{\pi i p j^{\prime} / q} \text { with } j^{\prime} \text { integral and } 1 \leqq j^{\prime}<q / 2 .
\end{aligned}
$$

For even $q$, setting $j=q / 2$ makes $e^{\pi i p j / q}$ equal to $i^{p}$, while for $j \neq q / 2$ we have the same fact that both $e^{\pi i p j / q}$ and $e^{\pi i p(q-j) / q}$ have negative imaginary parts or both have positive imaginary parts. Accordingly,

$$
(-i)^{\alpha(p, q)}= \begin{cases}(-1)^{N(p, q)}, & \text { for } p \equiv 1(\bmod 4), q \text { even, } \\ i(-1)^{N(p, q)+1}, & \text { for } p \equiv 3(\bmod 4), q \text { even. }\end{cases}
$$


Hence, from (4.8) we can conclude

(4.10) $f(-i)=\left\{\begin{array}{lr}(-1)^{N(p, q)} e^{-\pi i(q-1) / 4} q^{1 / 2}, & \text { for } q \text { even, } p \equiv 1(\bmod 4), \\ (-1)^{N(p, q)} i e^{-\pi i(q-1) / 4} q^{1 / 2}, & \text { for } q \text { even, } p \equiv 3(\bmod 4), \\ (-1)^{N(p, q)} e^{-\pi i(q-1) / 4} q^{1 / 2}, & \text { for } q \text { odd. }\end{array}\right.$

Another property of $f(\xi)$ is

$$
a_{j}=a_{q-j-1}(-1)^{(p-1)(q-1) / 2}, \quad j=1,2, \cdots, q-1 .
$$

In fact, the roots of $f(\xi)$ are simple and contain the reciprocal of each of them, whence

$$
\xi^{q-1} f\left(\xi^{-1}\right)=(-1)^{(p-1)(q-1) / 2} f(\xi)
$$

5. Real independence and semi-independence. The following definitions are variants of the notion of linear independence; they are introduced in order to simplify the formal part of our computations.

Definition. $n$ complex-valued functions $F_{1}(x), \cdots, F_{n}(x)$ on a range of the variable $x$ shall be called "really independent" on this range if the relation

$$
R\left\{\sum_{\nu=1}^{n} A_{\nu} F_{\nu}(x)\right\}=0
$$

with complex coefficients $A_{\nu}$ implies

$$
A_{1}=A_{2}=\cdots=A_{n}=0 \text {. }
$$

If and only if the $F_{\nu}(x)$ are really independent, the $2 n$ functions $R F_{\nu}(x)$ and $I F_{\nu}(x)$ are linearly independent in the ordinary sense employing real coefficients.

LEMMA. Let $F_{1}(x), \cdots, F_{n}(x)$ be really independent. Then a relation with complex coefficients $a_{\nu}, b_{\nu}$,

$$
R\left\{\sum_{1}^{n}\left(a_{\nu} F_{\nu}(x)+b_{\nu} \bar{F}_{\nu}(x)\right)\right\}=0
$$

implies

$$
a_{\nu}+b_{\nu}=0, \quad \quad \nu=1, \cdots, n \text {. }
$$

In fact,

$$
0=R\left\{\sum\left(a_{\nu} F_{\nu}(x)+b_{\nu} \bar{F}_{\nu}(x)\right)\right\} \equiv R\left\{\sum\left(a_{\nu}+b_{\nu}\right) F_{\nu}(x)\right\} .
$$

This identity shows that (5.1) follows from (5.2), no matter whether the $F_{\nu}$ are really independent or not. 
Definition. If $F_{1}(x), \cdots, F_{n}(x)$ are complex, $F(x)$ real, and such that

$$
R F_{1}(x), \cdots, R F_{n}(x), I F_{1}(x), \cdots, I F_{n}(x), F(x)
$$

are linearly independent, we shall call $F_{1}(x), \cdots, F_{n}(x), F(x)$ "really semi-independent."

Lemma. $A$ relation for complex coefficients $a_{\nu}, b_{\nu}, a$,

$$
\begin{aligned}
a_{\nu}+b_{\nu} & =0, \\
a+\bar{a} & =0,
\end{aligned}
$$

implies

$$
R\left\{\sum_{\nu=1}^{n}\left(a_{\nu} F_{\nu}(x)+b_{\nu} \bar{F}_{\nu}(x)\right)+a F(x)\right\}=0 .
$$

Conversely, (5.4) implies (5.3) if the $F_{\nu}(x)$ and $F(x)$ are really semiindependent.

Notation. In order to simplify the printing, we shall of ten make use of the following notation. Let $\gamma_{1}$ and $\gamma_{2}$ be two complex quantities. We write

$$
\gamma_{1} \cong \gamma_{2},
$$

in order to express that $\gamma_{1}$ and $\gamma_{2}$ are complex conjugate.

6. Integration of (3.4) for $E(z) \equiv 0$. Writing (3.4) in the form

$$
\prod_{j=1}^{q}\left(d / d z-\sigma_{j}\right) W(z)=0
$$

where

$$
\sigma_{i}=i / \epsilon^{j}=i e^{\pi i p j / q}
$$$$
j=1, \cdots, q,
$$

we obtain

$$
W(z)=\sum_{j=1}^{q} c_{j} e^{\sigma_{j} x}
$$

Note that

$$
\sigma_{j} \cong \sigma_{q-i}, \quad j=1, \cdots, q-1,
$$

whence

$$
e^{\sigma j x} \cong e^{\sigma q-i x}
$$


for real $x$. For odd $q$ the $e^{\sigma j x}(j=q, q-1, \cdots, q-(q-1) / 2=(q+1) / 2)$ are $(q+1) / 2$ really independent functions, for even $q$ the $e^{\sigma x}$ $(j=q, q-1, \cdots, q / 2+1)$ and $e^{\sigma / 2^{x}}$ are $q / 2+1$ really semi-independent functions. Both statements follow from the nonvanishing of the Wronskian of $e^{\sigma_{1} x}, \cdots, e^{\sigma_{q^{x}}}$, which in turn is a consequence of the nonvanishing of the Vandermondian of the $q$ distinct numbers $\sigma_{1}, \cdots, \sigma_{q}$. In fact a linear form

$$
R\left\{\sum_{1}^{n}\left(A_{\nu} e^{\sigma_{\nu} x}+B_{\nu} e^{\varepsilon_{\nu} x}\right)\right\}
$$

where $s_{\nu}=\bar{\sigma}_{\nu}$, can be written as

$$
(1 / 2) \cdot \sum_{1}^{n}\left(\left(A_{\nu}+\bar{B}_{\nu}\right) e^{\sigma_{\nu} x}+\left(\bar{A}_{\nu}+B_{\nu}\right) e^{s_{\nu} x}\right) .
$$

The $c_{j}$ are determined by the boundary conditions (2.1) and (2.3). We find

$$
0=R\{i d W / d z-W\}=R\left\{\sum_{1}^{q}\left(i \sigma_{j}-1\right) c_{j} e^{\sigma_{j} x}\right\}, \text { on } y=0,
$$

and

$$
0=R\left\{i \epsilon^{1 / 2} d W / d z\right\}=R\left\{\sum_{1}^{q} i \epsilon^{1 / 2} \sigma_{j} c_{j} e^{\sigma_{j z}}\right\}, \quad \text { on } \quad z=|z| \epsilon^{1 / 2}
$$

Applying the lemmas about real dependence resp. semi-independence in case $q$ is odd resp. even, we obtain as necessary and sufficient conditions for the validity of (6.4)

$$
c_{j}\left(i \sigma_{j}-1\right) \cong-c_{q-j}\left(i \sigma_{q-j}-1\right), \quad j=1, \cdots, q-1 .
$$

Note that, for $j=q$, we have $i \sigma_{q}-1=0$. In order to exploit (6.5), observe that by (6.2)

$$
\sigma_{q-j+1} \epsilon^{1 / 2} \cong \sigma_{j} \epsilon^{1 / 2}, \quad j=1, \cdots, q .
$$

Hence (6.5) implies

$$
i \epsilon^{1 / 2} \sigma_{q-j+1} c_{q-j+1} \cong-i \epsilon^{1 / 2} \sigma_{j} c_{j},
$$

which reduces to

$$
\epsilon \sigma_{q-j+1} c_{q-j+1}=\sigma_{q-j} \bar{c}_{j}
$$

or

$$
c_{q-j+1}=\bar{c}_{j}, \quad j=1, \cdots, q .
$$


The equations (6.6) and (6.9) determine the $c$, but for a real factor independent of $j$. We conclude directly

$$
c_{q-j}=c_{j}\left(i \sigma_{j}-1\right) /\left(i \sigma_{j}+1\right), \quad j=1, \cdots, q-1,
$$

or

$$
c_{j+1}=c_{j}\left(i \sigma_{j}-1\right) /\left(i \sigma_{j}+1\right), \quad j=1, \cdots, q-1 .
$$

The equations (6.11) are recursion formulae. They do not exhaust the implications of (6.6) and (6.9). Let $q$ be even, and apply (6.10) with $j=q / 2$ and $(6.2)$ :

$$
\bar{c}_{q / 2}=c_{q / 2}\left(1+\epsilon^{-q / 2}\right) /\left(\epsilon^{-q / 2}-1\right) .
$$

Now $\epsilon^{-q / 2}=e^{\pi i p / 2}$ and $p$ is odd. Hence

$$
\bar{c}_{q / 2}=c_{q / 2} i^{-p},
$$

for $q$ even.

Let $q$ be odd, and put $j=(q+1) / 2$ in (6.9):

$$
\bar{c}_{(q+1) / 2}=c_{(q+1) / 2}, \text { or } c_{(q+1) / 2} \text { is real for } q \text { odd. }
$$

Formulae (6.12) resp. (6.13) together with (6.11) show that the $c_{j}$ are determined but for a constant real factor.

To compute the $c_{j}$, we observe that they are related to the coeffcients $a_{j-1}$ of $f(\xi)$ in a simple manner. In fact, we had (4.5),

$$
a_{j}=a_{j-1} i\left(1+\epsilon^{j}\right) /\left(1-\epsilon^{j}\right),
$$

and (6.11) which can be written

$$
c_{j+1}=c_{j}\left(1+\epsilon^{j}\right) /\left(1-\epsilon^{j}\right), \quad j=1, \cdots, q-1 .
$$

Hence

$$
c_{j+1} / a_{j}=-i c_{j} / a_{j-1}, \quad j=1, \cdots, q-1,
$$

and

$$
c_{j}=a_{j-1}(-i)^{j} d,
$$$$
j=1, \cdots, q,
$$

where $d$ does not depend on $j$. The absolute value of $d$ is irrelevant, but its argument is of importance. Take $q$ even and apply (6.12); we find

$$
c_{q / 2}=e^{\pi i p / 4} r,
$$

with a real factor $r$. Substitute this value of $c_{q / 2}$ in the formula (6.14) for $j=q / 2$ and remember that all the coefficients $a_{j}$ are real. We find

$$
d=e^{\pi i(p+q) / 4},
$$

for $q$ even. 
Next take $q$ odd. Then, by (6.13), (6.14),

$$
(-i)^{(q+1) / 2} d=\text { real, }
$$

and

$$
d=i^{(q+1) / 2},
$$

for $q$ odd.

From (6.14), (4.1), (4.10) we compute

$$
\sum_{1}^{q} c_{j}=-i f(-i) d
$$

(6.17) $\sum_{1}^{q} c_{j}=\left\{\begin{array}{llr}(-1)^{N(p, q)} q^{1 / 2}, & \text { for } q \text { odd, } \\ (-1)^{N(p, q)+(p-1) / 4} q^{1 / 2}, & \text { for } & q \text { even, } p \equiv 1(\bmod 4), \\ (-1)^{N(p, q)+(p+1) / 4} q^{1 / 2}, & \text { for } & q \text { even, } p \equiv 3(\bmod 4) .\end{array}\right.$

Furthermore, by (6.14) and (4.11),

$$
c_{q}= \begin{cases}(-i)^{(q-1) / 2}, & \text { for } q \text { odd, } \\ i e^{-\pi i(p+q) / 4}, & \text { for } q \text { even. }\end{cases}
$$

Accordingly, our solution $W(z)$, designated henceforth by $W_{0}(z)$, is (6.19) $W_{0}(z)=\left\{\begin{array}{rr}(-i)^{(q-1) / 2} e^{-i z}+i^{(q+1) / 2} \sum_{j=1}^{q-1}(-i)^{j} a_{j} \exp \left[i z e^{i \pi j p / q}\right], \\ & \text { if } q \text { odd, } \\ i e^{-\pi i(p+q) / 4} e^{-i z}+e^{\pi i(p+q) / 4} \sum_{j=1}^{q-1}(-i)^{j} a_{j} \exp \left[i z e^{i \pi j p / q}\right], & \text { if } q \text { even, }\end{array}\right.$

with

$$
a_{j}=\prod_{n=1}^{j} \cot (n p \pi / 2 q), \quad a_{0}=1
$$

In order to see that the coefficients $c_{2}$ actually satisfy (6.6) and (6.9), we notice that these relations contain at most $2 q-1$ linearly independent real equations, while on the other hand their consequences (6.11) and (6.12) resp. (6.13) are precisely $2 q-1$ linearly independent relations. Thus the number of linearly independent equations (6.6) and (6.9) is $2 q-1$, and they follow from (6.11) and (6.12) resp. (6.13).

7. Behavior of $W_{0}(z)$ for $z \rightarrow \infty$. For the applicability of (6.19) to the wave problem the behavior of $W_{0}(z)$ at infinity is of importance. 
Now the moduli of the terms under the sum in (6.19) tend to zero or infinity as $z=x \rightarrow \infty$, according to whether $R\left\{i e^{i \pi p j / q}\right\}<0$ or $>0$. Furthermore, it is readily seen that the modulus of the sum cannot remain finite unless all terms tend to zero. Now let $1<p<2 q$ and consider the angles

$$
p \pi / q, 2 p \pi / q, \cdots,(q-1) p \pi / q,
$$

and their conventional representation in the unit circle. Among them there is at least one whose second side lies in the third or fourth quadrant. This is evident if $p>q$, because $p \pi / q$ has this property. For $p<q$ we remember that there are at most $(q-1)$ positions available in the first and second quadrants, namely those of

$$
\pi / q, 2 \pi / q, \cdots,(q-1) \pi / q .
$$

If our contention were wrong, they would have to be filled each by a member of the set (7.1), since no two of them can occupy the same position by virtue of the fact that $p$ and $q$ are relatively prime. In particular there would be one, say $k p \pi / q$, which occupies the same position as $(q-1) \pi / q$. Hence $k p=q-1+n q$ with even $n$. Here $k \neq q-1$ since otherwise $p-1$ becomes divisible by $q$ in contradiction to $p<q$, $q>1$. But now $(k+1) p \pi / q$ is among the angles (7.1) and its second side is identical with that of

$$
(q-1+p) \pi / q=(1+(p-1) / q) \pi,
$$

which lies in the third or fourth quadrant.

From the foregoing we gather that, for $1<p<2 q$, there exists a $j$ with $1 \leqq j \leqq q-1$ for which $R\left\{i e^{i \pi j p / q}\right\}>0$. Accordingly $W_{0}(x)$ does not remain finite as $x \rightarrow \infty$ in contradiction of the condition $5^{\prime}$ of $\$ 2$.

For $p=1$, however, $R\left\{i e^{i \pi / p / q}\right\}<0$ for $j=1,2, \cdots, q-1$, and $W_{0}(x)$ remains finite as $x \rightarrow \infty$. Hence $R W_{0}(z)$ represents a standing wave for $p=1$, it is free of singularities and behaves for $z \rightarrow \infty$ like a simple standing wave in water of infinite depth. More precisely,

$$
R W_{0}(z) \sim R\left\{e^{-\pi i(q-1) / 4} e^{-i z}\right\}, \quad \text { for } q \text { odd or even, }
$$

and the complex factor $e^{-\pi i(q-1) / 4}$ is decisive in the location of the nodes of this simple wave. These nodes lie at $x=(-(q-1) / 4+n) \pi$ with integral $n$. Accordingly, an increase of one unit in $q$ makes the nodes of the simple wave move one-eighth of a wave length toward shore, if only angles $\beta$ of the form $\pi / 2 q$ are considered.

The standing wave $R W_{0}(z)$ was given by E. T. Hanson. ${ }^{3}$ As we have proved, the angles $\beta$ of the form $\pi / 2 q$ between surface and floor of the

${ }^{3}$ Loc. cit. 
ocean are the only ones for which it will satisfy the condition of finiteness at infinity.

For the construction of a progressive wave, Hanson's standing wave furnishes only one component for $p=1$. For this construction requires the exhibition of a second standing wave of the same period which at large distance from the shore becomes a simple wave whose nodes lie midway between those of the first component.

Plan of the following investigation. The assumption $E(z)=$ const. underlying the preceding section is a special case of (3.4). More generally we can take

$$
E(z)=i z^{2 k q / p}, \quad k=0, \pm 1, \pm 2, \cdots .
$$

All these choices may be expected to lead to a solution $W(z)$ of (2.1) and (2.3), that is, to a $W(z)$ satisfying the boundary conditions. But, generally speaking, these $W(z)$ do not satisfy the condition of finiteness at infinity, and some have too large a singularity at the origin. Our plan is to obtain suitable superpositions of the various solutions which insure finiteness at infinity, indeed two independent such superpositions, so as to enable us to prescribe the location of the nodes of the simple standing wave to which they become asymptotically equal. It is remarkable that this procedure proves successful, in that the number of conditions to be satisfied is larger than the number of available constants. Consequently, it becomes necessary to investigate in detail the asymptotic behavior of the solutions to be constructed, and the algebraic character of the conditions which enforce finiteness at infinity.

The result will be that for all angles $\beta=\pi p / 2 q, 1 \leqq p<2 q, p$ odd, there exist two standing waves, $\phi_{1}$ and $\phi_{2}$, one which remains finite at the shore (and whose derivatives of first order (the velocity components) remain finite), the other having a logarithmic singularity at $z=0$ (resp. having a velocity which becomes infinite like $1 /|z|$ ).

8. Properties of exponential integrals. Unfortunately, the construction of waves runs in a similar, but not identical, pattern when $p \equiv 1(\bmod 4)$ and when $p \equiv 3(\bmod 4)$. This necessitates two parallel sets of formulae for certain parts of the following investigation.

We begin with $p \equiv 3(\bmod 4)$. Let $\alpha \geqq 0$ and consider the integral

$$
\int_{\alpha}^{\sigma_{j} z} e^{-t} t^{\lambda} d t
$$

where

$$
\lambda=(2 k q / p)-1, \quad k=0, \pm 1, \pm 2, \cdots
$$


The integral is a multi-valued function of $z$, but a single-valued function of $\log z$, once $\log \sigma_{j}$ and the path of integration on the Riemann surface of $\log z$ have been fixed. We shall take $\log z$ real for $z=x>0$ (on the surface) and set, generally,

$$
\zeta=\log z
$$

and

$$
\tau_{j}=\log \sigma_{j}
$$

with

$$
\tau_{j}=-p \pi i / 2+j p \pi i / q,
$$$$
j=1, \cdots, q .
$$

This choice of the $\tau_{j}$ has been made with a view to establishing for the $\tau_{j}$ the same relations of conjugation that hold for the $\sigma_{j}$. In fact, the analogues of (6.3) and (6.7) are

$$
\tau_{q-j}=p \pi i / 2-j p \pi i / q=\bar{\tau}_{j}, \quad 1 \leqq j<q,
$$

and

$$
\begin{array}{r}
\tau_{j}-p \pi i /(2 q) \cong p \pi i / 2-p(j-1 / 2) \pi i / q=\tau_{q-j+1}-p \pi i /(2 q), \\
1 \leqq j \leqq q .
\end{array}
$$

Accordingly, let the path of integration start at that point $\alpha$ which belongs to a real value of $\log \alpha$ and end at that point $\sigma_{j} z$ whose $\log$ is $\zeta+\tau_{j}$, and define

$$
L_{\lambda, \sigma j}(\zeta)=e^{\sigma j z} \int_{\alpha}^{\sigma_{j z} z} e^{-t t^{\lambda}} d t
$$

For real $\log z$, that is, for $z=x>0$ (on the surface), this function satisfies, because of (8.6) and (8.7), (8.3) and (8.4),

$$
L_{\lambda, \sigma_{j}}(\zeta) \cong L_{\lambda, \sigma q-j}(\zeta) \text {. }
$$

For $\zeta=\log z=$ real $-i p \pi / 2 q$, that is, for $z=r \epsilon^{1 / 2}, r>0$ (on the bottom),

$$
L_{\lambda, \sigma_{j}}(\zeta) \cong L_{\lambda, \sigma_{q-i+1}}(\zeta) \text {. }
$$

We find the differential equation

$$
(d / d z) L_{\lambda, \sigma_{j}}(\zeta)-\sigma_{j} L_{\lambda, \sigma_{j}}(\zeta)=\sigma_{j}^{\lambda+1} z^{\lambda}=z^{-1} e^{\left(\zeta+r_{j}\right) 2 k q / p},
$$

valid for all $\zeta$. Thus for real $\zeta, z=x>0$,

$$
(d / d z) L_{\lambda, \sigma_{j}}(\zeta)=\sigma_{j} L_{\lambda, \sigma_{j}}(\zeta)+x^{\lambda}(-1)^{k q},
$$

and for $\zeta=\operatorname{real}-i p \pi / 2 q, z=r \epsilon^{1 / 2}$, 


$$
(d / d z) L_{\lambda, \sigma_{j}}(\zeta)=\sigma_{j} L_{\lambda, \sigma_{j}}(\zeta)+r^{\lambda}(-1)^{k(q+1)} \epsilon^{-1 / 2} .
$$

Next take $p \equiv 1(\bmod 4)$. Set

$$
\tau_{j}=\log \left(-\sigma_{j}\right),
$$

where, again, (8.5) is assumed:

$$
\tau_{j}=-p \pi i / 2+j p \pi i / q, \quad j=1, \cdots, q .
$$

Then, we have the same relations of conjugations as before, namely (8.6) and (8.7). The definition paralleling (8.8) becomes

$$
L_{\lambda, \sigma_{j}}(\zeta)=e^{\sigma_{j} z} \int_{\alpha}^{-\sigma_{j} z} e^{t t^{\lambda}} d t
$$

It introduces a single-valued function of $\zeta$ for $\alpha \geqq 0, \log \alpha$ real, and $\lambda=(2 k q / p)-1$ as above. We conclude the same relations (8.9) and $(8.10)$ of conjugation for the new definition $\left(8.8^{\prime}\right)$. Furthermore, the differential equation corresponding to $(8.11)$ becomes

$$
(d / d z) L_{\lambda, \sigma_{j}}(\zeta)=\sigma_{j} L_{\lambda, \sigma_{j}}(\zeta)+\left(-\sigma_{j}\right)^{\lambda+1} z^{\lambda}
$$

and its special cases corresponding to surface and bottom are again the equations (8.12) and (8.13).

Now consider, no matter what $p$, the function $W_{\lambda}(z)$ defined by

$$
W_{\lambda}(z) \equiv \sum_{j=1}^{q} c_{j} L_{\lambda, \sigma j}(\zeta)
$$

We gather from (8.12) that on the surface where $z=x>0$

$$
i(d / d z) W_{\lambda}(z)-W_{\lambda}(z)=\sum_{1}^{q} c_{j}\left(i \sigma_{j}-1\right) L_{\lambda, \sigma_{j}}(\zeta)+x^{\lambda}(-1)^{k q} i \sum_{1}^{q} c_{j},
$$

and on the bottom where $z=r \epsilon^{1 / 2}, r>0$,

$$
i \epsilon^{1 / 2}(d / d z) W_{\lambda}(z)=\sum_{j=1}^{q} i \epsilon^{1 / 2} c_{j} \sigma_{j} L_{\lambda, \sigma_{j}}(\zeta)+r^{\lambda}(-1)^{k(q+1)} i \sum_{1}^{q} c_{j} .
$$

Now, since $\sum_{1}^{a} c_{j}$ is real by (6.17), we have on the surface

$$
R\left\{i(d / d z) W_{\lambda}(z)-W_{\lambda}(z)\right\}=R \sum_{1}^{q} c_{j}\left(i \sigma_{j}-1\right) L_{\lambda, \sigma_{j}}(\zeta),
$$

and on the bottom

$$
R\left\{i \epsilon^{1 / 2}(d / d z) W_{\lambda}(z)\right\}=R \sum_{1}^{q} i \epsilon^{1 / 2} c_{j} \sigma_{j} L_{\lambda, \sigma j}(\zeta) .
$$


But then the equations (6.6) and (6.8) are sufficient, because of the conjugation relations (8.9) and (8.10), to insure

$$
\begin{array}{rlr}
R\left\{\left(i \frac{d}{d z}-1\right) W_{\lambda}(z)\right\} & =0 & \text { on the surface, } \\
R\left\{i \epsilon^{1 / 2}(d / d z) W_{\lambda}(z)\right\} & =0 & \text { on the bottom. }
\end{array}
$$

This shows that $R W_{\lambda}(z)$ is a harmonic function satisfying the boundary conditions on surface and bottom.

It is of interest that the functions $L_{\lambda, \sigma_{j}}(\zeta)$ are linearly independent as functions of $z$ for $j=1, \cdots, q$; this implies their real independence or semi-independence (see \$5) on the surface as well as on the bottom. But since this fact is of no influence on the explicit formulae which we shall give for the waves, we suppress a proof thereof.

9. Construction of a second standing wave for $p=1$. We are now in a position to complete the construction of the progressive wave for $p=1$ by establishing a second standing wave which behaves differently at infinity from Hanson's standing wave given by (6.19).

This second standing wave is

$$
R W_{-1}(z)=R \sum_{j=1}^{q} c_{j} L_{-1, \sigma_{j}}(\zeta)
$$

To show this we study the behavior of $W_{-1}(z)$ for large $|z|$ in $S_{\beta}$.

Notice that a change of the value of the positive lower limit $\alpha$ in the definition $\left(8.8^{\prime}\right)$ of the integral affects $W_{-1}(z)$ merely by adding a real constant times $W_{0}(z)$ to it. For simplicity's sake take $\alpha=1$. We have

$$
L_{-1, \sigma_{j}}(\zeta)=e^{\sigma_{j z} z} \int_{1}^{-\sigma_{j} z} e^{t} t^{-1} d t=\int_{1}^{-\sigma_{j} z} e^{t+\sigma_{j} z t^{-1}} d t,
$$

with the values (8.5)

$$
\log -\sigma_{j}=\tau_{j}=-\pi i / 2+j \pi i / q, \quad j=1, \cdots, q .
$$

Hence the arguments of $-\sigma_{j} z$ lie between $-\pi i / 2+\pi i / 2 q$ and $\pi i / 2$, as $z$ varies in the sector $S_{\beta}$. Thus the variable of integration may be restricted to the half-plane $R(t) \geqq 0$.

With this in mind, let $\gamma=\gamma_{1}+\gamma_{2} i, \gamma_{1} \geqq 0,|\gamma| \geqq 1$. There exists an $M>0$ such that

$$
\left|\int_{1}^{\gamma} e^{t-\gamma} t^{-1} d t\right| \leqq M .
$$


For we have

$$
\begin{aligned}
\int_{1}^{\gamma} e^{t-\gamma} t^{-1} d t & =\left.e^{t-\gamma t^{-1}}\right|_{1} ^{\gamma}+\int_{1}^{\gamma} e^{t-\gamma t^{-2}} d t \\
& =\gamma^{-1}-e^{1-\gamma}+\int_{1}^{\gamma} e^{t-\gamma} t^{-2} d t
\end{aligned}
$$

and

$$
\left|\int_{1}^{\gamma} e^{t-\gamma} t^{-2} d t\right| \leqq e \int_{1}^{\gamma}|t|^{-2}|d t| \leqq e\left(1-|\gamma|^{-1}+\pi / 2\right) .
$$

This latter estimate is obtained by making the path of integration proceed first on the unit circle and then on a ray through the origin and $\gamma$. Consequently, (9.2) holds with any $M$ for which

$$
M \geqq|\gamma|^{-1}+e^{1-\gamma_{1}}+e(1+\pi / 2),
$$

for example, for $M=1+e(2+\pi / 2)$.

Hence $W_{-1}(z)$ remains bounded in the sector $S_{\beta}$ as long as $|z| \geqq 1$. Moreover, as $z=x \rightarrow \infty, L_{-1, \sigma_{j}}(\zeta) \rightarrow 0$ for $j=1, \cdots, q-1$; for from (9.3) with $-\sigma_{j} x=\gamma_{1}+\gamma_{2} i$ we gather $\gamma_{1}>0$ and

$$
\begin{aligned}
\left|\int_{1}^{\gamma} e^{t-\gamma} t^{-1} d t\right| \leqq & \gamma^{-1}-e^{1-\gamma}+\left(\int_{1}^{\gamma_{1} / 2}+\int_{\gamma_{1} / 2}^{\gamma}\right) e^{t-\gamma} t^{-2} d t \mid \\
\leqq & \left.\gamma\right|^{-1}+e^{1-\gamma_{1}}+e^{-\gamma_{1} / 2}\left(1-2 \gamma_{1}^{-1}\right) \\
& +\left|\gamma_{1}^{-1}-2 \gamma_{1}^{-1}\right| .
\end{aligned}
$$

The terms of the last expression tend individually to zero as $x \rightarrow \infty$. On the other hand, consider

$$
L_{-1, \sigma_{q}}(\zeta)=\int_{1}^{i x} e^{t-i x t^{-1}} d t
$$

as $x \rightarrow \infty$. By a deformation of the path of integration in the left halfplane we obtain in a familiar way

$$
\int_{1}^{i \infty} e^{t} t^{-1} d t=\int_{1}^{1-\infty} e^{t} t^{-1} d t+\pi i=r+\pi i,
$$

where the sign $\int^{\prime}$ denotes the Cauchy principal value of the real integral. Accordingly,

$$
W_{-1}(x)-c_{Q} e^{-i x} \int_{1}^{i x} e^{t} t^{-1} d t \rightarrow 0 \text { as } x \rightarrow \infty
$$


this leads to

$$
W_{-1}(x)-c_{q} e^{-i x}(r+\pi i) \rightarrow 0 \text { as } x \rightarrow \infty,
$$

since the integral (9.4) converges. Thus $W_{-1}(x)-r W_{0}(x)$ behaves like $c_{q} e^{-i x} \pi i$ as $x \rightarrow \infty$, while, by (6.18) and (6.19), $W_{0}(z)$ behaves like $c_{q} e^{-i x}$. Obviously the nodes of $R\left\{c_{q} e^{-i x}\right\}$ lie midway between those of $R\left\{c_{q} e^{-i x} \pi i\right\}$.

Near the origin we find, since $\sum c_{j}$ is real, in view of (6.17), that

$$
R W_{-1}(z) \sim q^{1 / 2} \log |z| .
$$

10. Asymptotic behavior of exponential integrals. We proceed to the study of the asymptotic behavior of the functions $L_{\lambda, \sigma j}(\zeta)$. For simplicity of notation, we set, for $p \equiv 3(\bmod 4)$,

$$
\gamma=z \sigma_{j}, \quad H_{\lambda}(\gamma)=e^{\gamma} \int_{\alpha}^{\gamma} e^{-t t^{\lambda}} d t
$$

where now $k$ is supposed not to be divisible by $p$ and

$$
\lambda=(2 k q / p)-1,
$$$$
k=1,2, \cdots \text {. }
$$

Furthermore $1<p<2 q, \alpha=0$. Since $\lambda>0$, integration by parts yields

$$
\begin{aligned}
\int_{0}^{\gamma} e^{-t t^{\lambda}} d t= & -\left.e^{-t t^{\lambda}}\right|_{0} ^{\gamma}+\lambda \int_{0}^{\gamma} e^{-t t^{\lambda-1}} d t, \\
H_{\lambda}(\gamma)= & -\gamma^{\lambda}-\lambda \gamma^{\lambda-1}-\cdots-\lambda(\lambda-1) \cdots(\lambda-s+1) \gamma^{\lambda-\bullet} \\
& +\lambda(\lambda-1) \cdots(\lambda-s) e^{\gamma} \int_{0}^{\gamma} e^{-t t^{\lambda-\sigma-1} d t,}
\end{aligned}
$$

a formula which holds for all integral $s$ with $0<s<\lambda$. In particular, we shall take the value $s=[\lambda]$, defined as the largest integer less than $\lambda$. We find

$$
\begin{aligned}
W_{\lambda}(z) \equiv & \sum_{1}^{q} c_{j} L_{\lambda, \sigma_{j}}(\zeta) \\
= & \sum c_{j} \lambda_{j}^{\lambda} z^{\lambda}\left(-1-\lambda\left(\sigma_{j} z\right)^{-1}-\cdots-\lambda \cdots(\lambda-s+1)\left(\sigma_{j} z\right)^{-\sigma}\right) \\
& +\lambda(\lambda-1) \cdots(\lambda-s) \sum c_{j} e^{\sigma_{j} z} \int_{0}^{\sigma_{j} z} e^{-t t^{\lambda-s-1} d t .}
\end{aligned}
$$

Now

$$
\sum c_{j} \sigma_{j}^{\lambda}=\sum c_{j} e^{\tau_{j} \lambda}=\sum c_{j} e^{\tau_{j} 2 k q / p_{\sigma_{j}}}{ }^{-1},
$$


and by (6.14) and (8.5) this becomes

$$
\begin{aligned}
\sum c_{j} \sigma_{j}^{\lambda} & =d \sum_{1}^{q} a_{j-1}(-i)^{j} e^{(-p / 2+j p / q) \pi i \cdot 2 k q / p} \sigma_{j}^{-1} \\
& =d \sum a_{j-1}(-i)^{j-1} \epsilon^{j}(-1)^{k q+1}=f(-i \epsilon) d \epsilon(-1)^{k q+1}=0,
\end{aligned}
$$

for by the lemma of $\$ 4$ the roots of $f(\xi)$ are $-i \epsilon, \cdots,-i \epsilon^{q-1}$.

Similarly $\sum_{1}^{a} c_{j} \sigma_{j}^{\lambda-1}=f\left(-i \epsilon^{2}\right) \cdot$ const. $=0$, where the constant denotes a number which does not depend on the index $j$ of summation. Proceeding in a like manner, we get

$$
\sum_{1}^{q} c_{j} \sigma_{j}^{\lambda \rightarrow}=f\left(-i \epsilon^{a+1}\right) \cdot \text { const. }=0
$$

for all $s>0$ with $s \leqq q-2$. This is compatible with $s=[\lambda]=[2 k q / p-1]$ for all $k$ with

$$
1 \leqq k \leqq(p-1) / 2
$$

In fact, then

$$
\begin{aligned}
{[2 k q / p] } & \leqq[(p-1) q / p] \leqq[q-q / p] \leqq q-1, \\
{[\lambda] } & =[2 k q / p-1] \leqq q-2 .
\end{aligned}
$$

For $k$ satisfying (10.4) we conclude from (10.2)

$$
W_{\lambda}(z)=W_{-1+2 k q / p}(z)
$$

Set

$$
=\Gamma(2 k q / p)(\Gamma(2 k q / p-[2 k q / p]))^{-1} \sum_{1}^{q} c_{j} L_{-1+2 k q / p-[2 k q / p]}(\zeta) .
$$

By (8.15), $W_{k}^{*}$ satisfies the boundary conditions on surface and bottom.

In order to study the behavior of $W_{k}^{*}(z)$ for $z=x \rightarrow \infty$ and for $z=r \epsilon^{1 / 2}, r \rightarrow \infty$, consider one of the integrals on the right of (10.5) (it will suffice to consider only the first of the following alternatives in case $p<q)$ :

$$
e^{\sigma_{j} x} \int_{0}^{\sigma_{j} x} e^{-t} t^{\lambda-[\lambda]-1} d t \text { resp. } e^{\sigma_{i} r \epsilon^{1 / 2}} \int_{0}^{\sigma_{j} r \epsilon^{1 / 2}} e^{-t t^{\lambda-[\lambda]-1} d t .}
$$

Suppose first that $j$ is such that

(10.7) $\pi / 2 \geqq-i \tau_{j} \geqq-\pi / 2$ resp. $\pi / 2 \geqq-i \tau_{j}-p \pi / 2 q \geqq-\pi / 2$.

Then 


$$
\lim _{x \rightarrow \infty} \int_{0}^{\sigma_{j} x} e^{-t} t^{\lambda-[\lambda]-1} d t=\Gamma(\lambda-[\lambda])
$$

resp.

$$
\lim _{r \rightarrow \infty} \int_{0}^{\sigma_{j} r \mathbf{e}^{1 / 2}} e^{-t} t^{\lambda-[\lambda]-1} d t=\Gamma(\lambda-[\lambda]),
$$

whence

$$
e^{\sigma_{j} x} \int_{0}^{\sigma_{j} x} e^{-t} t^{\lambda-[\lambda]-1} d t=\Gamma(\lambda-[\lambda]) e^{\sigma_{j} x}-e^{\sigma_{j} x} \int_{\sigma_{j} x}^{\infty} e^{-t} t^{\lambda-[\lambda]-1} d t
$$

resp.

$$
e^{\sigma_{j} \mathrm{e}^{1 / 2}} \int_{0}^{\sigma_{j} \boldsymbol{\epsilon} \mathrm{1} / 2} e^{-t} t^{\lambda-[\lambda]-1} d t
$$

Here

$$
=\Gamma(\lambda-[\lambda]) e^{\sigma_{j} r \epsilon^{1 / 2}}-e^{\sigma_{j} r \epsilon^{1 / 2}} \int_{\sigma j r \epsilon^{1 / 2}}^{\infty} e^{-t} t^{\lambda-[\lambda]-1} d t
$$

$$
\begin{aligned}
\left|e^{\sigma_{j} x} \int_{\sigma j x}^{\infty}\right| & =\left|\int_{0}^{\infty} e^{-u}\right| u+\left.\sigma_{j} x\right|^{\lambda-[\lambda]-1} d u \mid \\
& \leqq\left|\sigma_{j} x\right|^{\lambda-[\lambda]-1} \int_{0}^{\infty} e^{-u} d u \rightarrow 0,
\end{aligned}
$$

resp.

$$
\left|e^{\sigma_{i} r \epsilon^{1 / 2}} \int_{\sigma j r \epsilon^{1 / 2}}^{\infty}\right| \rightarrow 0,
$$

since $\lambda-[\lambda]-1<0$. Thus

$$
e^{\sigma_{j} x}\left(\int_{0}^{\sigma_{j} x} e^{-t} t^{\lambda-[\lambda]-1} d t-\Gamma(\lambda-[\lambda])\right) \underset{x \rightarrow \infty}{\rightarrow} 0, \text { resp. }
$$

$$
e^{\sigma_{j} r \epsilon^{1 / 2}}\left(\int_{0}^{\sigma_{j} \epsilon^{1 / 2}} e^{-t} t^{\lambda-[\lambda]-1}-\Gamma(\lambda-[\lambda])\right) \underset{r \rightarrow \infty}{\rightarrow} 0,
$$

if (10.7) holds.

Observe that $\int_{0}^{\gamma} e^{-t} t^{\lambda-[\lambda]-1} d t$ is multiplied by $e^{2 \pi i \lambda}$ as $\log \gamma$ is increased by $2 \pi i$. Therefore we can generalize the previous result. Suppose $n_{j}$ defined equal to $n$, provided there exists an integral number $n$ such that 


$$
\begin{aligned}
& \text { either } n 2 \pi+\pi / 2 \geqq-i \tau_{j} \geqq n 2 \pi-\pi / 2 \\
& \text { or } \quad n 2 \pi+\pi / 2 \geqq-i \tau_{j}-p \pi /(2 q) \geqq n 2 \pi-\pi / 2 .
\end{aligned}
$$

(Notice that $p<2 q$ so that these two conditions never lead to different values of $n_{j}$.) Then

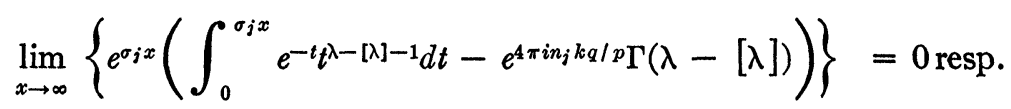

$$
\lim _{r \rightarrow \infty}\left\{e^{\sigma_{j} r e^{1 / 2}}\left(\int_{0}^{\sigma_{j} r e^{1 / 2}} e^{-t} t^{\lambda-[\lambda]-1} d t-e^{4 \pi i n_{j} k q / p} \Gamma(\lambda-[\lambda])\right)\right\}=0 .
$$

On the other hand, if one or both of the conditions (10.9) cannot be satisfied with integral $n$, but instead we have with some $n$

$$
\begin{aligned}
& n 2 \pi+\pi / 2<-i \tau_{j}<n 2 \pi+3 \pi / 2 \text { resp. } \\
& n 2 \pi+\pi / 2<-i \tau_{j}-p \pi / 2 q<n 2 \pi+3 \pi / 2,
\end{aligned}
$$

then we must have, for example,

hence

$$
\begin{aligned}
\left|e^{\sigma_{j} x} \int_{0}^{\sigma_{j} x}\right| & =\left|\int_{0}^{-\sigma_{j} x} e^{-u}\left(u+\sigma_{j} x\right)^{\lambda-[\lambda]-1} d u\right| \leqq\left|\int_{0}^{\infty}\right|+\left|\int_{-\sigma_{j}, x \mid}^{\infty}\right| \\
& \leqq|x|^{\lambda-[\lambda]-1}+\left|e^{\sigma_{j} x}\right| \Gamma(\lambda-[\lambda]) ;
\end{aligned}
$$

$$
\lim _{x \rightarrow \infty} e^{\sigma_{j} x} \int_{0}^{\sigma_{j} x} e^{-t t^{\lambda}-[\lambda]-1} d t=0, \text { resp. }
$$

$$
\lim _{r \rightarrow \infty} e^{\sigma_{j} r \mathrm{e}^{1 / 2}} \int_{0}^{\sigma_{j} r \mathrm{e}^{1 / 2}} e^{-t} t^{\lambda-[\lambda]-1} d t=0 .
$$

These estimates show that

$$
\begin{aligned}
\lim _{x \rightarrow \infty}\left\{W_{k}^{*}(x)-\sum_{j=1}^{q} c_{j} \delta_{j k} e^{\sigma_{j} x}\right\} & =0, \\
\lim _{r \rightarrow \infty}\left\{W_{k}^{*}\left(r \epsilon^{1 / 2}\right)-\sum_{1}^{q} c_{j} \delta_{j k} e^{\sigma_{j} r \epsilon^{1 / 2}}\right\} & =0,
\end{aligned}
$$

where $\delta_{j k}$ stands for

$$
\delta_{j k}= \begin{cases}e^{4 \pi i n j k q / p} & \text { if }(10.9) \text { holds, } \\ 0 & \text { if (10.11) holds }\end{cases}
$$

(the value of $n_{j}$ in the second case is immaterial for the validity of 
(10.12) because of the vanishing of the exponential factor). The possible values of $n_{j}$ in (10.9) are

(10.14) $\quad n_{i}=(p+1) / 4 ;(p-3) / 4, \cdots, 0,-1, \cdots,-(p-3) / 4$.

The first of these occurs only for $j=q$, that is, $n_{q}=(p+1) / 4$. All the other possible values of $n_{j}$ must be successive integers since two successive terms $-i \tau_{j}-p \pi / 2 q,-i \tau_{j}$ or $-i \tau_{j},-i \tau_{j+1}-p \pi / 2 q$ differ by $p \pi / 2 q<\pi$. On the other hand, the smallest value of $n_{j}$ occurs either in $n_{j} 2 \pi-\pi / 2 \leqq-i \tau_{j} \leqq n_{j} 2 \pi+\pi / 2$ or in $n_{j} 2 \pi-\pi / 2 \leqq-i \tau_{j}-p \pi / 2 q$ $\leqq n_{j} 2 \pi+\pi / 2$ when this $n_{j}$ is the smallest integer following that one, $n^{\prime}$, which would occur if $j$ could become equal to zero; this would give

$$
2 n^{\prime} \pi+\pi / 2=-p \pi / 2, \quad n^{\prime}=-(p+1) / 4 .
$$

The alternative in the relations (10.9) to (10.14) is dictated by the possibility that $p>q$. Had we demanded that $p<q$, then the Phragmén-Lindelöf principle would have permitted us to fix our attention solely on the surface, as was explained in $\$ 2$.

Next we give the parallel developments for $p \equiv 1(\bmod 4)$. Integrate by parts for $\lambda>0$ :

$$
\begin{aligned}
\int_{0}^{-\gamma} e^{t} t^{\lambda} d t= & \left.e^{t t^{\lambda}}\right|_{0} ^{-\gamma}-\lambda \int_{0}^{-\gamma} e^{t t^{\lambda-1}} d t=\cdots \\
e^{\gamma} \int_{0}^{-\gamma} e^{t} t^{\lambda} d t= & (-\gamma)^{\lambda}-\lambda(-\gamma)^{\lambda-1}+\cdots \\
& +(-1)^{s}(-\gamma)^{\lambda-s} \lambda(\lambda-1) \cdots(\lambda-s+1) \\
& +(-1)^{s+1} e^{\gamma} \int_{0}^{-\gamma} e^{t t^{\lambda-\sigma-1}} d t \lambda(\lambda-1) \cdots(\lambda-s),
\end{aligned}
$$

where $s=[\lambda]$. Then, by $\left(8.8^{\prime}\right)$,

$$
\begin{aligned}
W_{\lambda}(z) \equiv \sum_{j=1}^{q} c_{j} L_{\lambda, \sigma_{j}}(\zeta) \\
=\sum_{1}^{q}\left[c _ { j } ( - \sigma _ { j } z ) ^ { \lambda } \left(1+\lambda\left(\sigma_{j} z\right)^{-1}+\cdots\right.\right. \\
\left.\quad+\lambda \cdots(\lambda-s+1)\left(\sigma_{j} z\right)^{-s}\right) \\
\left.\quad+c_{j}(-1)^{s+1} e^{\sigma_{j} z} \int_{0}^{-\sigma_{j} z} e^{t} t^{\lambda-s-1} d t \lambda \cdots(\lambda-s)\right]
\end{aligned}
$$

Now 


$$
\begin{aligned}
& \sum_{1}^{q} c_{j}\left(-\sigma_{j}\right)^{\lambda}=\sum_{1}^{q} c_{j} e^{r j \lambda}=d \sum_{1}^{q} a_{j-1}(-i)^{j} e^{(-p / 2+j p / q) 2 k q \pi i / p}\left(-\sigma_{j}\right)^{-1} \\
& =\text { const. } \sum a_{j-1}(-i \epsilon)^{j-1}=\text { const. } f(-i \epsilon)=0 \text {, } \\
& \sum_{1}^{q} c_{j}\left(-\sigma_{j}\right)^{\lambda-s}=\text { const. } \sum_{1}^{q} a_{j-1}(-i)^{j-1} \epsilon^{(j-1)(8+1)} \\
& \text { = const. } f\left(-i \epsilon^{\varepsilon+1}\right)=0 \text {, }
\end{aligned}
$$

since (10.3) and (10.4) apply again. Hence, for $k=1,2, \cdots,(p-1) / 2$, $\lambda=(2 k q / p)-1$,

$$
\begin{aligned}
W_{k}^{*}(z) & \equiv(\Gamma(\lambda+1))^{-1} W_{\lambda}(z) \\
& =(-1)^{[2 k q / p]}(\Gamma(\lambda-[\lambda]))^{-1} \sum_{j=1}^{q} c_{j} L_{\lambda-[\lambda]-1, \sigma j}(\zeta)
\end{aligned}
$$

satisfies the boundary conditions on surface and bottom.

For large $x$, resp. large $r$, consider

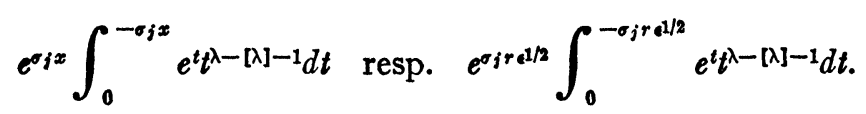

For such $j$ as make

$$
-\pi / 2<-i \tau_{j}<\pi / 2 \text { resp. }-\pi / 2<-i \tau_{j}-p \pi / 2 q<\pi / 2,
$$

we have $R\left(-\sigma_{j}\right)>0$, and, with $\gamma=-\sigma_{j} x$,

$$
\left(10.11 .1^{\prime}\right) \quad e^{-\gamma} \int_{0}^{\gamma} e^{t} t^{\lambda-[\lambda]-1} d t=\int_{0}^{\gamma} e^{t-\gamma} t^{\lambda-[\lambda]-1} d t \rightarrow 0,
$$

as $x \rightarrow \infty$; or resp. $\left(10.11 .1^{\prime}\right)$ holds with $\gamma=-\sigma_{j} r \epsilon^{1 / 2}$ and $R\left(-\sigma_{j} \epsilon^{1 / 2}\right)>0$ as $r \rightarrow \infty$. Similarly, $\left(10.11 .1^{\prime}\right)$ still holds if there is an integral $n$ for which

$$
\begin{aligned}
& -\pi / 2+2 n \pi<-i \tau_{j}<\pi / 2+2 n \pi \text { resp. } \\
& -\pi / 2+2 n \pi<-i \tau_{j}-p \pi / 2 q<\pi / 2+2 n \pi
\end{aligned}
$$

On the other hand, if there is an integral $n$ such that

$$
\begin{aligned}
& \pi / 2+2 n \pi \leqq-i \tau_{j} \leqq 3 \pi / 2+2 n \pi \quad \text { resp. } \\
& \pi / 2+2 n \pi \leqq-i \tau_{j}-p \pi / 2 q \leqq 3 \pi / 2+2 n \pi,
\end{aligned}
$$

then let $n_{j}$ be defined by $n_{j}=n$ in $\left(10.9^{\prime}\right)$. We have $R \gamma \leqq 0$, where

$$
\gamma=-\sigma_{j} x \quad \text { resp. } \gamma=-\sigma_{j} r e^{1 / 2},
$$




$$
\begin{aligned}
e^{-\gamma} \int_{0}^{\gamma} e^{t} t^{\lambda-[\lambda]-1} d t & =e^{-\gamma}\left(\int_{0}^{-\infty}+\int_{-\infty}^{\gamma}\right), \\
\int_{0}^{-\infty} e^{t} t^{\lambda-[\lambda]-1} d t & =-\int_{0}^{\infty} e^{-|t|}|t|^{\lambda-[\lambda]-1} d|t| e^{\pi i\left(1+2 n_{j}\right)(\lambda-[\lambda]-1)} \\
& =e^{\left(2 n_{j}+1\right) \pi i 2 k q / p}(-1)^{[2 k q / p]} \Gamma(\lambda-[\lambda]), \\
\left|e^{-\gamma} \int_{-\infty}^{\gamma} e^{t} t^{\lambda-[\lambda]-1} d t\right| & =\mid \int_{-\infty}^{\gamma} e^{t-\gamma t^{\lambda-[\lambda]-1} d t \mid} \\
& =\left|\int_{-\infty}^{0} e^{u}(u+\gamma)^{\lambda-[\lambda]-1} d u\right| \\
& \leqq|\gamma|^{\lambda-[\lambda]-1} \rightarrow 0,
\end{aligned}
$$

as $x \rightarrow \infty$ resp. $r \rightarrow \infty$.

Hence

$$
\begin{aligned}
\lim _{x \rightarrow \infty}\left[e^{\sigma j x} \int_{0}^{-\sigma_{j} x} e^{t} t^{\lambda-[\lambda]-1} d t\right. & \\
& \left.-(-1)^{[2 k q / p]} \Gamma(\lambda-[\lambda]) e^{\sigma j x} e^{\left(2 n_{j}+1\right) \pi i 2 k q / p}\right]=0,
\end{aligned}
$$

resp. a similar limit equation for $r \rightarrow \infty$, if $x \sigma_{j}$ is replaced by $r \sigma_{j} \epsilon^{1 / 2}$. Accordingly, by $\left(10.6^{\prime}\right)$

$$
\begin{aligned}
& \lim _{x \rightarrow \infty}\left\{W_{k}^{*}(x)-\sum_{1}^{q} c_{j} \delta_{j k} e^{\sigma_{j} x}\right\}=0, \text { resp. } \\
& \lim _{r \rightarrow \infty}\left\{W_{k}^{*}\left(r \epsilon^{1 / 2}\right)-\sum_{1}^{q} c_{j} \delta_{j k} e^{\sigma_{j} r \epsilon^{1 / 2}}\right\}=0,
\end{aligned}
$$

were now

$$
\delta_{j k}=\left\{\begin{array}{l}
e^{2 \pi i k q\left(2 n_{j}+1\right) / p} \\
0
\end{array}\right.
$$

if $\left(10.11^{\prime}\right)$ holds, otherwise.

Here the possible values of $n_{j}$ range from $(p-1) / 4$, occurring for $j=q$ when $-\sigma_{q}=i, \tau_{q}=p \pi / 2$, to $-(p-1) / 4$, occurring for the smallest $j$ for which $\left(10.9^{\prime}\right)$ holds. For this value exceeds by 1 the value $-(p+3) / 4$ which would occur if $j=0$ were admissible; for then

$$
3 \pi / 2+2 n^{\prime} \pi=-p \pi / 2, \quad n^{\prime}=-(p+3) \pi / 4 .
$$

11. Extension to the case $\lambda=-1, p \equiv 3(\bmod 4)$. If $k$ in $(8.2)$ is set equal to zero, $\alpha$ can no longer be chosen as zero if the integral (8.1) is to converge. We set $\alpha=1$ and study the asymptotic behavior 
of $L_{-1, \sigma_{j}}(\zeta)$ defined by

$$
L_{-1, \sigma j}(\zeta)= \begin{cases}e^{\sigma_{j} z} \int_{1}^{\sigma_{i} z} e^{-t} t^{-1} d t, & \text { for } p \equiv 3(\bmod 4), \\ e^{\sigma_{j} z} \int_{1}^{-\sigma_{j} z} e^{-t} t^{-1} d t, & \text { for } p \equiv 1(\bmod 4) .\end{cases}
$$

Here again the generalities about the multivalued character of the integral apply. If $\zeta$ increases by $2 \pi i, L_{-1, \sigma_{j}}(\zeta)$ changes in the following manner:

$$
L_{-1, \sigma j}(\zeta+2 \pi i)=L_{-1, \sigma j}(\zeta)+2 \pi i e^{\sigma j z} .
$$

Furthermore, there exists the conjugation

$$
L_{-1, \sigma_{j}}(\zeta) \cong L_{-1, s_{j}}(\xi)
$$

where $s_{j}=\bar{\sigma}_{j}$. Let $\gamma \neq 0,-\pi / 2 \leqq \arg \gamma \leqq \pi / 2, \log \gamma=\log |\gamma|+i \arg \gamma$. Then

$$
\begin{aligned}
e^{\gamma} \int_{1}^{\gamma} e^{-t} t^{-1} d t & =e^{\gamma}\left(\int_{1}^{\infty}-\int_{\gamma}^{\infty}\right) \\
& =e^{\gamma} \int_{1}^{\infty} e^{-t} t^{-1} d t-\int_{0}^{\infty} e^{-t}(t+\gamma)^{-1} d t
\end{aligned}
$$$$
\left|e^{\gamma} \int_{1}^{\gamma} e^{-t} t^{-1} d t-e^{\gamma} \int_{1}^{\infty} e^{-t} t^{-1} d t\right| \leqq|\gamma|^{-1}
$$$$
e^{\gamma} \int_{1}^{\gamma} e^{-t} t^{-1} d t-e^{\gamma} \int_{1}^{\infty} e^{-t} t^{-1} d t \rightarrow 0
$$

as $\gamma \rightarrow \infty,|\arg \gamma| \leqq \pi / 2$. But if $\pi / 2+\delta<\arg \gamma<3 \pi / 2-\delta, \delta>0$, consider $e^{\gamma} \int_{1}^{\gamma} e^{-t} t^{-1} d t$ where the integral is extended along a path leading in the upper half-plane from 1 to $\gamma /|\gamma|$ and thence to $\gamma$ on a straight line. We have, with $\kappa=\gamma /|\gamma|=-\kappa_{1}+i \kappa_{2}, \kappa_{1}>\sin \delta>0$,

$$
\begin{aligned}
\left|e^{\gamma} \int_{1}^{\gamma} e^{-t} t^{-1} d t\right| & \leqq\left|e^{\gamma} \int_{1}^{\kappa} e^{-t} t^{-1} d t\right|+\left|\int_{\kappa}^{\gamma} e^{-(t-\gamma)} t^{-1} d t\right| \\
\left|\int_{\kappa}^{\gamma} e^{-(t-\gamma)} t^{-1} d t\right| & \leqq\left|\int_{1}^{|\gamma|} e^{-\kappa(t-|\gamma|)} t^{-1} d t\right| \\
& \leqq\left|\int_{1}^{|\gamma| / 2}\right|+\left|\int_{|\gamma| / 2}^{|\gamma|}\right| \\
& \leqq e^{-\kappa_{1}|\gamma| / 2} \log (|\gamma| / 2)+2\left(\kappa_{1}|\gamma|\right)^{-1} .
\end{aligned}
$$

Hence $e^{\gamma} \int_{1}^{\gamma} e^{-t} t^{-1} d t \rightarrow 0$ as $\pi / 2+\delta \leqq \arg \gamma \leqq 3 \pi / 2-\delta$ and $|\gamma| \rightarrow \infty$. Con- 
sequently, for $n_{j}$ as defined by (10.9) and $\zeta=\log x$ real resp. $\zeta=\log$. $-i p \pi / 2 q, \log r$ real,

$$
L_{-1, \sigma_{j}}(\zeta)-2 \pi i n_{j} e^{\sigma_{j} x}-e^{\sigma_{j} x} \int_{1}^{\infty} e^{-t t^{-1}} d t \rightarrow 0, \quad \text { if } R \sigma_{j} \geqq 0,
$$

resp.

$$
L_{-1, \sigma_{j}}(\zeta)-2 \pi i n_{j} e^{\sigma_{j} r^{e / 1 / 2}}-e^{\sigma_{j r} e^{1 / 2}} \int_{1}^{\infty} e^{-t} t^{-1} d t \rightarrow 0,
$$

if $R\left(\sigma_{j} \epsilon^{1 / 2}\right) \geqq 0$,

while always

$$
\lim _{x \rightarrow \infty} L_{-1, \sigma_{j}}(\zeta)=0, \quad \text { if } R \sigma_{j}<0, \zeta=\log x
$$

resp.

$$
\lim _{x \rightarrow \infty} L_{-1, \sigma_{j}}(\zeta)=0, \quad \text { if } R\left(\sigma_{j} \epsilon^{1 / 2}\right)<0, \zeta=\log \left(r \epsilon^{1 / 2}\right) .
$$

The formulae (11.6) and (11.7) lead to corresponding relations for

$$
W_{-1}(\zeta) \equiv \sum_{1}^{q} c_{i} L_{-1, \sigma_{j}}(\zeta)
$$

12. Extension to the case $\lambda=-1, p \equiv 1(\bmod 4)$. Let $R-\sigma_{j}>0$. Then with some integral $n$ and $\gamma=-\sigma_{j} x, R \gamma>0$,

$$
e^{-\gamma} \int_{1}^{\gamma} e^{t} t^{-1} d t=2 \pi i n e^{-\gamma}+\int_{1}^{\gamma} e^{t-\gamma} t^{-1} d t
$$

where the last integral can be taken along a straight line and

$$
\begin{aligned}
\left|\int_{1}^{\gamma} e^{t-\gamma t-1} d t\right| & \leqq\left|\int_{1}^{\gamma|| \gamma \mid}\right|+\left|\int_{\gamma /|\gamma|}^{\gamma}\right| \\
& \leqq e^{-R \gamma} \cdot \text { const. }+e^{-R \gamma} \int_{1}^{R \gamma} e^{t t^{-1}} d t .
\end{aligned}
$$

Consequently, if $\gamma$ tends to $\infty$ on a ray through the origin which lies in $R \gamma>0$, we have $e^{-\gamma} \int_{1}^{\gamma} e^{t} t^{-1} d t \rightarrow 0$. Now suppose $n_{j}$ defined by $\left(10.9^{\prime}\right)$, that is,

$$
\pi / 2+2 n_{j} \pi \leqq-\tau_{j} i \leqq 3 \pi / 2+2 n_{j} \pi
$$

resp.

$$
\pi / 2+2 n_{j} \pi \leqq-\tau_{j} i-p \pi i / 2 q \leqq 3 \pi / 2+2 n_{j} \pi .
$$


We have in the first alternative

$$
e^{\sigma j x} \int_{1}^{-\sigma j x} e^{t} t^{-1} d t=e^{\sigma j x} \int_{1}^{-\infty} e^{t} t^{-1} d t+e^{\sigma j x} \int_{-\infty}^{-\sigma_{j} x} e^{t-1} d t,
$$

where

$$
\int_{1}^{-\infty} e^{t} t^{-1} d t=\int_{1}^{1-\infty} e^{t} t^{-1} d t+\left(2 n_{j}+1\right) \pi i
$$

with $\int^{\prime}$ understood as Cauchy's principal value, and where the path of $\int_{-\infty}^{-\sigma_{j} x}$ remains in the negative half-plane. Now as $x \rightarrow \infty$,

$$
\begin{aligned}
\left|e^{\sigma_{j} x} \int_{-\infty}^{-\sigma_{j} x} e^{t} t^{-1} d t\right| & =\left|\int_{-\infty}^{-\sigma_{j} x} e^{t+\sigma_{j} x t^{-1}} d t\right| \\
& =\left|\int_{-\infty}^{0} e^{t}\left(t-\sigma_{j} x\right)^{-1} d t\right| \leqq x^{-1} \rightarrow 0
\end{aligned}
$$

so that

$$
\begin{aligned}
(12.1) e^{\sigma_{j} x}\left[\int_{1}^{-\sigma_{j} x} e^{t} t^{-1} d t-\left(2 n_{j}+1\right) \pi i-\int_{1}^{1-\infty} e^{t} t^{-1} d t\right] & \rightarrow 0, \\
& \text { for } x \rightarrow \infty, R \sigma_{j} \geqq 0 .
\end{aligned}
$$

For the second alternative of $\left(10.9^{\prime}\right)$ we have similarly

$$
\begin{array}{r}
e^{\sigma j r \epsilon^{1 / 2}}\left[\int_{1}^{-\sigma_{j} \epsilon^{1 / 2}} e^{t} t^{-1} d t-\left(2 n_{j}+1\right) \pi i-\int_{1}^{1-\infty} e^{t} t^{-1} d t\right] \rightarrow 0, \\
\text { for } r \rightarrow \infty, R \sigma_{j} \epsilon^{1 / 2} \geqq 0 .
\end{array}
$$

13. Construction of the finite standing wave. It is now possible to exhibit a solution of our boundary problem in the form

$$
\Omega_{0}(z)=W_{0}(z)-\sum_{k=1}^{(p-1) / 2} A_{k} W_{k}^{*}(z) ;
$$

$\Omega_{0}(z)$ remains bounded as $z$ tends to infinity on the surface and bottom of $S_{\beta}$, and remains bounded as $z \rightarrow 0$. Moreover, the derivative $d \Omega_{0}(z) / d z$ will also remain bounded near $z=0$ as is obvious from the definition (6.19) of $W_{0}(z)$ and the definitions (10.6) resp. (10.6') and (10.2) resp. $\left(10.2^{\prime}\right)$. Our problem is to determine the $A_{k}$ in such a way as to eliminate by superposition the various infinities introduced for large $|z|$ by each of the summands in (13.1). It suffices if this can 
be done on the surface for $p<q$, and on the surface and bottom for $q<p<2 q$. Let $p \equiv 3(\bmod 4)$. Fix the attention first on the surface. The estimates (10.10) and (10.12) lead to the equation

$$
\lim _{x \rightarrow \infty}\left[\Omega_{0}(x)-\sum_{j}^{\prime} c_{j} e^{\sigma j x}\left(1-\sum_{k=1}^{(p-1) / 2} \delta_{j k} A_{k}\right)\right]=0,
$$

where it is necessary to extend the sum $\sum^{\prime}$ only over those values $j$ for which the first alternative of (10.9) applies. Now suppose that for those values of $n_{j}$ for which the first alternative (10.9) applies, but with the exception of $j=q$, we can solve the system of linear equations

$$
\sum_{k=1}^{(p-1) / 2} \delta_{j k} A_{k}=1, \quad j \neq q,
$$

in the unknowns $A_{k}$. Then (13.2) reduces to

$$
\lim _{x \rightarrow \infty}\left[\Omega_{0}(x)-c_{q} e^{-i x}\left(1-\sum_{k=1}^{(p-1) / 2} \delta_{q k} A_{k}\right)\right]=0 .
$$

In order that $\Omega_{0}(x)$ should behave like a simple wave at infinity, we shall have to show in addition that

$$
-1+\sum_{k=1}^{(p-1) / 2} \delta_{q k} A_{k} \neq 0 .
$$

It follows directly that $\Omega_{0}(x)$ stays bounded also on the bottom if the $A_{k}$ satisfy the system of equations (13.3).

Now (10.14) shows that (13.3) represents exactly $(p-1) / 2$ distinct equations, namely

$$
\sum_{k=1}^{(p-1) / 2} A_{k} e^{4 \pi i k n q / p}=1, \quad n=-(p-3) / 4, \cdots,+(p-3) / 4 .
$$

A similar analysis in the case $p \equiv 1(\bmod 4)$ shows the corresponding equations to be

$$
\begin{aligned}
& \sum_{k=1}^{(p-1) / 2} A_{k} e^{2 \pi i k q(2 n+1) / p}=1, \\
& n=-(p-1) / 4, \cdots,(p-5) / 4 .
\end{aligned}
$$

We proceed to give the values of the $A_{k}$ explicitly. There occurs in this deduction a remarkable analogy to the computation of the coefficients $a_{i}$ of the polynomial $f(\xi)$ introduced in $\$ 4$, with the role of $p$ and $q$ interchanged. Consider the polynomial of degree $(p-1) / 2$, 


$$
F(\xi)=\sum_{0}^{(p-1) / 2} A_{k} \xi^{k}, \quad \text { with } A_{0}=-1,
$$

which vanishes at the $(p-1) / 2$ distinct points

$$
\begin{aligned}
& \xi=e^{4 \pi i n q / p}, \\
n & =-(p-3) / 4, \cdots,(p-3) / 4, \text { for } p \equiv 3,
\end{aligned}
$$

resp.

$$
\begin{gathered}
\xi=e^{2 \pi i q(2 n+1) / p}, \\
n=-(p-1) / 4, \cdots,(p-5) / 4, \text { for } p \equiv 1(\bmod 4) .
\end{gathered}
$$

We have

$$
\begin{array}{ll}
F(\xi)=\prod_{|n| \leqq(p-3) / 4}\left(\xi-\eta^{n}\right), & \eta=e^{4 \pi i q / p}, \text { for } p \equiv 3(\bmod 4), \\
F(\xi)=-\prod_{n \geq-(p-1) / 4}^{(p-5) / 4}\left(\xi-\eta^{2 n+1}\right), & \eta=e^{2 \pi i q / p}, \text { for } p \equiv 1(\bmod 4) .
\end{array}
$$

Evidently (13.6) resp. (13.6') hold. To test (13.5) we write for $p \equiv 3(\bmod 4)$

$$
\text { (13.9) }-1+\sum_{k=1}^{(p-1) / 2} \delta_{q k} A_{k}=F\left(e^{4 \pi i q(p+1) /(4 p)}\right)=F\left(e^{(p+1) q \pi i / p}\right) \neq 0
$$

since $e^{(p+1) q \times i / p}$ coincides with none of the roots (13.7). Similarly for $p \equiv 1(\bmod 4)$

$$
\begin{aligned}
-1+\sum_{1}^{(p-1) / 2} \delta_{q k} A_{k} & =F\left(e^{2 \pi i(q / p)(2(p-1) / 4+1)}\right) \\
& =F\left(e^{(p+1) q \pi i / p)}\right.
\end{aligned}
$$

which does not vanish for a similar reason. It is easy to evaluate the $A_{k}$ explicitly. We have for $p \equiv 3(\bmod 4)$

$$
\left(\xi-\eta^{(p-3) / 4}\right) F(\eta \xi)=\eta^{(p-1) / 2} F(\xi)\left(\xi-\eta^{-(p+1) / 4}\right),
$$

whence

$$
\sum_{0}^{(p-1) / 2} A_{k} \xi^{k+1}\left(\eta^{k}-\eta^{(p-1) / 2}\right)=\sum_{0}^{(p-1) / 2} A_{k} \xi^{k}\left(\eta^{k}-1\right) \eta^{(p-3) / 4}
$$

But

$$
\eta^{p / 2}=1, \quad \eta^{p / 4}=(-1)^{q}
$$

and 


$$
A_{k-1}=(-1)^{q} A_{k}\left(\eta^{k-8 / 4}-\eta^{-8 / 4}\right) /\left(\eta^{k-1}-\eta^{-1 / 2}\right)
$$

so that

$$
\begin{aligned}
A_{k} & =(-1)^{q} A_{k-1}(\sin (2 k-1) q \pi / p) / \sin 2 k q \pi / p \\
& =(-1)^{k q+1} \prod_{\nu=1}^{k} \frac{\sin (2 \nu-1) q \pi / p}{\sin 2 \nu q \pi / p} ; \quad A_{0}=-1 .
\end{aligned}
$$

Similarly for $p \equiv 1(\bmod 4)$

$$
\begin{aligned}
\left(\xi-\eta^{(p-1) / 2}\right) F\left(\eta^{2} \xi\right) & =\eta^{p-1}\left(\xi-\eta^{-(p-1) / 2}\right) F(\xi), \\
\sum A_{k} \xi^{k+1}\left(\eta^{2 k}-\eta^{p-1}\right) & =\sum A_{k} \xi^{k}\left(\eta^{(p-1) / 2} \eta^{2 k}-\eta^{(p-1) / 2}\right) .
\end{aligned}
$$

But here

$$
\begin{aligned}
\eta^{p}=1, \quad \eta^{p / 2} & =(-1)^{q}, \quad A_{k}=(-1)^{q} A_{k-1} \eta^{-1 / 2}\left(\eta^{2 k-1}-1\right) /\left(\eta^{2 k}-1\right) \\
A_{k} & =(-1)^{q} A_{k-1}(\sin (2 k-1) q \pi / p) / \sin 2 k q \pi / p
\end{aligned}
$$

which again leads to the result (13.10).

We find jointly for $p \equiv 3$ and $p \equiv 1(\bmod 4)$ from $(13.8)$

$$
(-1)^{(p+1) / 2} F\left(e^{\pi i q(p+1) / p}\right)=\left(e^{\pi i q(p+1) / p}-e^{\pi i q(p-3) / p}\right) \cdots,
$$

and by elementary simplification

$$
(-1)^{(p+1) / 2} F\left(e^{\pi i q(p+1) / p}\right)=e^{\pi i q\left(p^{2}-1\right) /(4 p)} \prod_{1}^{(p-1) / 2}\left(e^{2 \pi i q k / p}-e^{-2 \pi i q k / p}\right),
$$

and by a reasoning similar to that employed in $\S 4$,

$$
(-1)^{(p+1) / 2} F\left(e^{\pi i q(p+1) / p}\right)=e^{\pi i q\left(p^{2}-1\right) / 4 p} i^{(p-1) / 2}(-1)^{N(2 q, p)} p^{1 / 2} .
$$

Here $N(2 q, p)$ represents again the number of terms in the set $e^{2 \pi i q / p}, e^{4 \pi i q / p}, \cdots, e^{\pi i(p-1) q / p}$ whose imaginary part is negative. Accordingly, by (13.4) and (13.9) resp. (13.9'),

$$
\lim _{x \rightarrow \infty}\left[\Omega_{0}(x)+c_{q} e^{-i x} F\left(e^{(p+1) q \pi i / p}\right)\right]=0,
$$

or

$$
\begin{aligned}
0=\lim _{x \rightarrow \infty}[ & \Omega_{0}(x)+c_{q} e^{-i x} e^{\pi i q\left(p^{2}-1\right) / 4 p} \\
& \left.\cdot i^{(p-1) / 2}(-1)^{N(2 q, p)+(p+1) / 2} p^{1 / 2}\right] .
\end{aligned}
$$

14. Construction of a second standing wave for $p \equiv 3(\bmod 4)$. Second interpolating function $G(\xi)$. Consider the polynomial

$$
G(\xi)=\sum_{1}^{(p-1) / 2} B_{k} \xi^{k}
$$


where

$$
\begin{aligned}
B_{k} & =-i\left(A_{k} / 2\right)\left(\eta^{k}-\eta^{-k}\right) /\left(\eta^{k}+\eta^{-k}-2\right) \\
& =-\left(A_{k} / 2\right) \cot 2 \pi q k / p
\end{aligned}
$$

is real. We have

$$
B_{k}=-i\left(A_{k} / 2\right)\left(\left(1-\eta^{k}\right)^{-1}-\left(1-\eta^{-k}\right)^{-1}\right),
$$

(14.3) $G(\xi \eta)-G(\xi)=\sum B_{k} \xi^{k}\left(\eta^{k}-1\right)=(i / 2)\left(\sum_{1}^{(p-1) / 2} \xi^{k} A_{k}\left(1+\eta^{k}\right)\right)$

$$
=(i / 2)(F(\xi)+F(\xi \eta)+2) \text {, }
$$

since $A_{0}=-1$. Also

$$
G(1)=\sum_{1}^{(p-1) / 2} B_{k},
$$

whence, by (13.8) and (14.3),

(14.4) $G\left(\eta^{n}\right)=i n+\sum_{1}^{(p-1) / 2} B_{k}$, for $n=-(p-3) / 4, \cdots,(p-3) / 4$, and

(14.5) $G\left(\eta^{(p+1) / 4}\right)=i(p+1) / 4+(i / 2) F\left(\eta^{(p+1) / 4}\right)+\sum B_{k}$.

Thus the $B_{k}$ are real numbers for which, with $\delta_{j k}$ from (10.13),

$$
\sum B_{k} \delta_{j k}=i n_{j}+\sum B_{k} \text {, }
$$
if (10.9) with $j \neq q$,

and

$$
\sum B_{k} \delta_{q k}=i(p+1) / 4+(i / 2) F\left(\eta^{(p+1) / 4}\right)+\sum B_{k} .
$$

Set

$$
\begin{aligned}
\pi \Omega_{-1}(z) \equiv W_{-1}(z)-2 \pi \sum_{1}^{(p-1) / 2} W_{k}^{*}(z) B_{k} \\
+W_{0}(z)\left(2 \pi \sum B_{k}-\int_{1}^{\infty} e^{t} t^{-1} d t\right) .
\end{aligned}
$$

By (14.7), (14.6), (11.6), (11.10) we have the limit relation

$$
\begin{aligned}
\lim _{x \rightarrow \infty}\left[-\pi \Omega_{-1}(x)+2 \pi i \sum_{j}^{\prime} c_{j} n_{j} e^{\sigma j x}\right. & -2 \pi \sum_{j}^{\prime} \sum_{k=1}^{(p-1) / 2} B_{k} \delta_{j k} c_{j} e^{\sigma_{j} x} \\
& \left.+\sum_{j}^{\prime} 2 \pi c_{j} \sum B_{k} e^{\sigma_{j} x}\right]=0
\end{aligned}
$$


where the sum $\sum^{\prime}$ extends over all $j$ with (10.9). This immediately yields

$$
\lim _{x \rightarrow \infty}\left[-\pi \Omega_{-1}(x)-\pi i c_{q} F\left(\eta^{(p+1) / 4}\right) e^{-i x}\right]=0 .
$$

Compare (14.8) with the limit formula (13.12) for $\Omega_{0}(x)$. Clearly the nodes of $\Omega_{-1}(x)$ lie asymptotically midway between those of $\Omega_{0}(x)$. In a similar manner it is seen that $\Omega_{-1}(z) \rightarrow 0$ on the bottom of $S_{\beta}$.

For $z \rightarrow 0, \Omega_{-1}(z)$ has a logarithmic singularity while $\Omega_{0}(z)$ remains finite. Furthermore, $\Omega_{-1}(z)$ satisfies the differential equations on surface and bottom since it is a linear combination with real coefficients of functions having this property. Thus $R \Omega_{-1}(z)$ is the velocity potential of a second standing wave and

$$
\cos t R \Omega_{0}(z)-\sin t R \Omega_{-1}(z)
$$

is the time-depending velocity potential of a progressive wave, advancing toward the shore $z=0$, which at large distances from shore is a simple progressive wave of wave length $2 \pi$. We observe that it has a logarithmic singularity at $z=0$.

15. Same for $p \equiv 1(\bmod 4)$. Set, as before

$$
G(\xi)=\sum_{1}^{(p-1) / 2} B_{k} \xi^{k},
$$

with

$$
\begin{aligned}
B_{k} & =-(i / 2) A_{k}\left(\left(1-\eta^{2 k}\right)^{-1}-\left(1-\eta^{-2 k}\right)^{-1}\right) \\
& =-\left(A_{k} / 2\right) \cot (2 \pi k q / p)
\end{aligned}
$$

where now

$$
\eta=e^{2 \pi i q / p}
$$

We find

$$
G\left(\xi \eta^{2}\right)-G(\xi)=(i / 2)\left(F(\xi)+F\left(\xi \eta^{2}\right)+2\right),
$$

since $A_{0}=-1$. Also

(15.2) $G\left(\eta^{2 n+1}\right)=G(\eta)+i n, \quad n=-(p-1) / 4, \cdots,+(p-5) / 4$,

(15.3) $G\left(\eta^{(p+1) / 2}\right)=G(\eta)+i(p-1) / 4+(i / 2) F\left(\eta^{(p+1) / 2}\right)$.

Now, by (15.1),

$$
G(\eta)-G\left(\eta^{-1}\right)=i,
$$

since $F(\eta)=F\left(\eta^{-1}\right)=0$. Hence, because $B_{k}$ is real, 


$$
G(\eta)=i / 2+\text { real }
$$

Thus (15.2), (15.3) become

$$
\begin{aligned}
& G\left(\eta^{2 n+1}\right)= i(n+1 / 2)+R G(\eta), \\
& n=-(p-1) / 4, \cdots,(p-5) / 4, \\
& G\left(\eta^{(p+1) / 2}\right)=i(p+1) / 4+(i / 2) F\left(\eta^{(p+1) / 2}\right)+R G(\eta) .
\end{aligned}
$$

Accordingly

$$
\sum B_{k} \delta_{j k}=i\left(n_{j}+1 / 2\right)+R G(\eta),
$$

if $\left(10.9^{\prime}\right)$ holds with $j \neq q$,

$$
\sum B_{k} \delta_{q k}=i(p+1) / 4+(i / 2) F\left(\eta^{(p+1) / 2}\right)+R G(\eta) .
$$

Set

$$
\begin{aligned}
\pi \Omega_{-1}(z)= & W_{-1}(z)-2 \pi \sum W_{k}^{*}(z) B_{k} \\
& +W_{0}(z)\left(2 \pi R G(\eta)-\int_{1}^{1-\infty} e^{-t} t^{-1} d t\right)
\end{aligned}
$$

Then

$$
\begin{aligned}
0=\lim _{x \rightarrow \infty} & {\left[-\pi \Omega_{-1}(x)+2 \pi i \sum_{j}^{\prime} c_{j}\left(2 n_{j}+1\right) e^{\sigma j x}-2 \pi \sum_{j}^{\prime} c_{j} e^{\sigma j x} \sum_{k} B_{k} \delta_{j k}\right.} \\
& \left.+\left(2 \pi R G(\eta)-\int_{1}^{\prime-\infty} e^{t} t^{-1} d t\right) \sum^{\prime} c_{j} e^{\sigma j x}\right]
\end{aligned}
$$

Consequently

$$
0=\lim _{x \rightarrow \infty}\left[-\pi \Omega_{-1}(x)-\pi i c_{q} F\left(e^{\pi i(p+1) q / p}\right) e^{-i x}\right] .
$$

The same conclusions about the behavior of $\Omega_{-1}(z)$ on the bottom and near $z=0$, and about the asymptotic distribution of the nodes of $R \Omega_{0}(x)$ and $R \Omega_{-1}(x)$, hold as did for $p \equiv 3(\bmod 4)$. Again

$$
\cos t R \Omega_{0}(z)-\sin t R \Omega_{-1}(z)
$$

is the velocity potential of a progressive wave advancing toward shore which at large distance from shore becomes a simple wave of length $2 \pi$ and which has a logarithmic singularity at the shore.

16. About the continuity of the standing wave as function of the angle $p \pi / 2 q$ and the connection with the quadratic reciprocity law of number theory. Let us inspect the asymptotic location of the surface nodes of the finite standing wave $\Omega_{0}(z)$ as $z=x \rightarrow \infty$. On entering the 
value of $c_{q}$ from (6.18) in (13.13) we find that $\Omega_{0}(x)$ differs infinitely little from

$$
\begin{array}{cc}
(-i)^{(q-1) / 2+(p-1) / 2}(-1)^{N(2 q / p)} e^{\pi i\left(p^{2}-1\right) q / 4 p} e^{-i x} p^{1 / 2}, & \text { if } q \text { odd, } \\
(-i)^{(p-1) / 2} i e^{-\pi i(p+q) / 4+\pi i\left(p^{2}-1\right) q / 4 p}(-1)^{N(2 q, p)} e^{-i x} p^{1 / 2}, & \text { if } q \text { even. }
\end{array}
$$

Thus the nodes of $R \Omega_{0}(x)$ come to lie asymptotically at the points $x=\pi / 2+m \pi+q p \pi / 4-q \pi / 4 p-\pi(q+p-2) / 4$, with $m$ integral for $q$ odd, $x=m \pi+q p \pi / 4-q \pi / 4 p-\pi(1+q) / 4$, with $m$ integral for $q$ even. If the oddness of $p$ is utilized, it is seen that the nodes lie asymptotically at

$$
x=m \pi-\pi / 4-q \pi / 4 p,
$$

no matter whether $q$ is odd or even; for if $q$ is odd,

$$
q p-q-p \equiv-1(\bmod 4)
$$

and if $q$ is even

$$
q p-1-q \equiv-1(\bmod 4) .
$$

The result (16.1) is the generalization of a property announced at the end of $\S 7$ for the case $p=1$.

Next let us study the ratio of $\lim _{x \rightarrow \infty}\left(e^{i x} \Omega_{0}(x)\right)$ and $\Omega_{0}(0)$. By (13.1), (10.6)

$$
\Omega_{0}(0)=W_{0}(0)=\sum_{1}^{q} c_{i}
$$

as given by (6.17). Accordingly, by elementary simplification of the exponents, one obtains, for example, for odd $p$ and $q$ the formula

$$
\begin{aligned}
\lim _{x \rightarrow \infty}\left(e^{i x} \Omega_{0}(x)\right) / \Omega_{0}(0) & \\
= & (-1)^{N(2 q, p)+N(p, q)+(q p-p-q+1) / 4} e^{\pi i(1-q / p) / 4}(p / q)^{1 / 2} .
\end{aligned}
$$

Now suppose that the left-hand side depends continuously on the angle $p \pi / 2 q$ between surface and bottom. Then the same applies to the right-hand side, whence in particular the expression

$$
(-1)^{N(2 q, p)+N(p, q)+(q p-p-q+1) / 4}
$$

must be continuous in $p / q$ for $p$ and $q$ odd. But since it equals \pm 1 , this expression must have a constant value, which is determined as +1 by taking $p=q=1$ or $p=1, q=3$. Furthermore it is easily seen from the definition of $N(p, q)$ that, for odd $p$ and $q, N(p, q)=N(2 p, q)$. Thus the assumption of a continuous dependence of the finite stand- 
ing wave on $p / q$ implies

$$
(-1)^{N(2 q, p)+N(2 p, q)}=(-1)^{(p-1)(q-1) / 4} .
$$

This is, of course, the quadratic reciprocity law of number theory.

We shall not investigate here the above continuity question, nor shall we go here into a discussion of those problems which are immediately suggested by the above embedding of a number theoretical fact concerning two integers $p$ and $q$ into a continuous law concerning the quantity $p / q$.

University of California 